\title{
6 Sprachgebrauch und Ort
}

Karten sind, wie herausgearbeitet wurde, eine wichtige diagrammatische Grundfigur in der Linguistik (vgl. dazu Abschnitt 5.2). Sie werden eingesetzt, um Sprachdaten in einen geografischen Bezug zu bringen, sei das etwa, indem ein bestimmter Sprachgebrauch einen Ortsbezug der Sprecherin indiziert (damit z. B. ein Gegenstand der Varietätenlinguistik), oder indem bestimmte Ausdrücke Orte denotieren (und damit z. B. ein Untersuchungsgegenstand der Onomastik sind).

Die Fallstudie zu den Geokollokationen geht von einem diskurslinguistischen Forschungsinteresse (Spitzmüller/Warnke 2011; Bubenhofer 2018a) aus und verbindet die beiden Perspektiven: Sie zielt auf die Frage, welche diskursiv geprägten Konstruktionen von Welt existieren und wie diese untersucht werden können. So sind es Diskurse, die bestimmte Assoziationen zu Orten und Regionen prägen. Für jemanden aus Deutschland oder Österreich könnten zu den Begriffen Schweiz und Griechenland folgende Assoziationen ausgelöst werden:

- Schweiz: Banken, Schokolade, Steuerhinterziehung

- Griechenland: Finanzkrise, Urlaub, Flüchtlingskrise

Toponyme denotieren also nicht nur einen (mehr oder weniger klar) definierten Ort in der Welt, sondern sind auch mit einer diskursiv geprägten Semantik beladen. Diese Semantik ist aber eben abhängig von Diskursen, vom spezifischen Sprachgebrauch und damit automatisch auch von den geografischen Standorten, aber natürlich auch der sozialen, zeitlichen, thematischen etc. Standorten dieser Diskurse, ihrer Verortung. Jemand aus Griechenland oder aus der Schweiz hat womöglich andere Assoziationen zum selben Toponym.

Ziel der Analyse ist nun nicht eine kognitivistische Sicht auf die Assoziationen in den Köpfen einzelner Sprecherinnen und Sprecher, sondern die datengeleitete Analyse massenmedialer Diskurse. Dafür entwickelten wir eine einfache Operationalisierung des Phänomens diskursiv geprägter Semantik von Toponymen, indem wir Kollokatoren zu Toponymen, sogenannten „Geokollokationen, berechnen. ${ }^{57}$

57 Ergebnisse von Geokollokationsanalysen und auch erste Reflexionen zu den Visualisierungen dazu veröffentlichten wir bereits an verschiedenen Stellen (Bubenhofer et al. 2019; Bubenhofer 2014, 2018d). Die folgenden Ausführungen beruhen zu Teilen darauf, sind aber stark erweitert.

๖ Open Access. (c) 2020 Noah Bubenhofer, publiziert von De Gruyter. (c) BY Dieses Werk ist lizenziert unter der Commons Attribution 4.0 Lizenz.

https://doi.org/10.1515/9783110698732-006 


\subsection{Konzeption Geokollokationen}

\subsubsection{Operationalisierung}

Kollokationsprofile sind ein Mittel, die Semantik von Lexemen im Sinne einer Wortgebrauchssemantik, wie sie beispielsweise die linguistische Begriffsgeschichte vertritt (Busse et al. 1994), zu analysieren (Bubenhofer 2017). Während die lexematischen Bestandteile der Kollokationen grundsätzlich nicht eingeschränkt sind, beschränken sich Geokollokationen auf Kollokationen, bei denen die Basis ein Toponym ist.

Um die Frage nach einer diskursiv geprägten Weltsicht $\mathrm{zu}$ beantworten, sollen also alle statistisch signifikanten Kollokatoren zu den Toponymen in einem Korpus berechnet werden, wobei das Korpus den gewünschten Diskurs repräsentieren soll. Die damit verbundene diagrammatische Operation ist die Erstellung eines Indizes aller Fundstellen von Toponymen (diagrammatische Grundfigur: Liste) und die Überführung der Toponym-Types in den Vektorraum (diagrammatische Grundfigur: Vektor), indem diese durch ihr Kollokationsverhalten definiert werden. Da die Toponyme selbst nur sprachliche Zeichen sind, die (mehr oder weniger deutlich) auf geografische Orte referieren, kann diese Referenz in einem weiteren Schritt aufgelöst werden, indem das Denotat des Toponyms als Georeferenz in Form von Koordinaten auf einer Karte dargestellt wird. Damit findet eine weitere diagrammatische Transformation (diagrammatische Grundfigur: Karte) statt.

Für die Analysen wurden verschiedene Datengrundlagen verwendet, die je nach Untersuchungsinteresse ausgewählt worden sind. In den folgenden Ausführungen wird von Analysen in diesen zwei Korpora berichtet:

- Korpus Spiegel/Zeit: Das Korpus umfasst alle in der Zeit von 1946 bis 2016 erschienenen und in den jeweiligen Online-Archiven der gedruckten Ausgaben verfügbaren Artikel (705'576 Texte, 611 Mio. laufende Wortformen).

- Korpus Parlamentsprotokolle Bundestag Deutschland: Alle Protokolle der Legislatur 2009 bis 2013 des Deutschen Bundestags (363’000 Redebeiträge, 22 Mio. laufende Wortformen) auf der Basis des Korpus von Andreas Blätte (2013).

Die Korpusdaten sind mit Metadaten ausgezeichnet und mit Wortartklassen annotiert sowie lemmatisiert unter Verwendung des TreeTaggers (Schmid 1994). Die Wortartklassen-Annotation benutzt die Standardbibliothek für Deutsch des Treetaggers und diese folgt dem Stuttgart-Tübingen Tagset (STTS, Schiller et al. 1995). 


\subsubsection{Assoziationsmaß der Kollokationen und Toponymerkennung}

Für die weitere Analyse gilt es nun, die genaue Berechnung der Geokollokationen $\mathrm{zu}$ definieren und einen Weg zu finden, die Toponyme in den Daten zu finden. Was die Kollokationsberechnung angeht, schlägt Evert (2009) verschiedene statistische Maße vor, die sich auch für je unterschiedliche Zwecke eignen. Neben dem statistischen Maß, das die Assoziationsstärke zwischen den Kollokatoren ausdrückt, ist das sogenannte Fenster wichtig, also die maximal erlaubte Spannbreite zwischen den Kollokatoren. Während für viele linguistische Fragestellungen eher enge Fenster im Bereich von drei bis acht Wörtern benutzt werden und die Satzgrenze meistens nicht überschritten wird, ist für unsere Zwecke auch ein viel größeres Fenster, das maximal der Textlänge entspricht, denkbar. Denn von Interesse sind bei einem in einem Text aufgetauchten Toponym grundsätzlich alle im selben Text überzufällig häufig auftretenden Lexeme.

Als Assoziationsmaß verwenden wir Log-Likelihood (Dunning 1993; Evert 2009, 1235), was als stabiles und gleichzeitig unkompliziert $\mathrm{zu}$ berechnendes Maß für Kollokationen gilt und in der Diskurslinguistik häufig angewandt wird (Bubenhofer 2017).

Ausgangspunkt für die Berechnung der Kollokationen sind die Toponyme. Im Korpus sollen daher mit maschineller Methode alle Toponyme gefunden und annotiert werden. Zur Annotation von Toponymen kommen einerseits sogenannte Gazetteer-Verfahren oder kombinierte Verfahren zur Anwendung, die mit auf vorannotierten Trainingsdaten erzeugten statistischen Modellen arbeiten. Für die Studie wurde der Stanford Named Entity Recognizer (NER) (Finkel et al. 2005) in einer für das Deutsche adaptierten Version (Faruqui/Padó 2010) verwendet, der mit einem statistischen Modell zur Vorhersage von Toponymen arbeitet. Der Recognizer erkennt generell Eigennamen und unterteilt diese in die Klassen, wobei für unsere Zwecke nur die Ortsbezeichnungen (,LOC“ = Location) berücksichtigt werden.

Grundsätzlich ist die Klassifikation von Toponymen auch für Menschen nicht einfach, da es Übergangsbereiche und Zweifelsfälle gibt. Diese Zweifelsfälle oder Übergangsbereiche entstehen etwa bei der Entscheidung, ob eine Nominalgruppen wie „der höchste Berg Europas“ (Wimmer 1995, 374) oder eine Derivation wie „berlinerisch“ als Toponyme aufgefasst werden sollen. Der NER verwendet eine enge Definition von „Toponym“. Die berichteten Werte von Evaluationen des Parsers liegen bei Zeitungstexten bei F-Werten von knapp unter 80 (Faruqui/Padó 2010), wobei die Präzision mit 88 Prozentpunkten höher liegt als die Ausbeute (72,9). Dies bedeutet, dass es sich bei den erkannten Toponymen bei etwa $88 \%$ tatsächlich um Toponyme handelt und der Parser generell etwa $73 \%$ aller Toponyme findet. 


\subsubsection{Georeferenzierung}

In Tabelle 10 ist ein Ausschnitt aus einer so berechneten Liste von Geokollokationen abgebildet. Während die Analyse der Liste bezüglich eines bestimmten Toponyms, hier z. B. von „Sinai“, sehr gut in dieser Form machbar ist, wird eine Analyse über ein paar wenige Toponyme hinaus schwierig. Einerseits nimmt die Anzahl der Einträge rasch zu und ist damit weniger gut überblickbar, aber andererseits fällt stärker ins Gewicht, dass Bezüge zwischen den Toponymen schwer überschaubar werden, da man bei der Analyse unwillkürlich versucht, die Konzepte der Toponyme geografisch zu verorten. „Yunnan“ und „Sinai“ erscheinen in der Liste (je nach Sortierung) benachbart, verweisen aber auf eine Provinz im Südwesten Chinas bzw. eine Halbinsel Ägyptens. Um eine Sortierung der Liste nach geografischen Zusammenhängen zu ermöglichen, ist deshalb eine Georeferenzierung sinnvoll.

Tab. 10: Ausschnitt aus einer Liste von Geokollokationen: In der ersten Spalte steht das Toponym und in den letzten zwei Spalten der dazugehörige Kollokator mit Wortartklasse. Weitere Spalten: „freq“ = absolute Frequenz der Kollokation, „sig“ Signifikanzniveau p

\begin{tabular}{lllll}
\hline toponym & freq & sig & collocate & pos \\
\hline Yunnan & 31 & 0.0001 & Provinz & NN \\
Sinai & 27 & 0.0001 & töten & VVPP \\
Sinai & 32 & 0.0001 & werden & VAPP \\
Sinai & 36 & 0.0001 & Al-Arisch & NN \\
Sinai & 23 & 0.0001 & Islamisten & NN \\
Sinai & 54 & 0.0001 & Halbinsel & NN \\
Sinai & 24 & 0.0001 & Mann & NN \\
Sinai & 30 & 0.0001 & öffentlich & ADJA \\
Sinai & 21 & 0.0001 & Al-Arisch & ADJD \\
Sinai & 20 & 0.0001 & Demonstrant & NN \\
Sinai & 39 & 0.0001 & Norden & NN \\
Sinai & 24 & 0.0001 & Polizist & NN \\
Sinai & 25 & 0.0001 & Stadt & NN \\
Sinai & 29 & 0.0001 & ägyptisch & ADJA \\
Sinai & 26 & 0.0001 & Extremist & NN \\
\hline
\end{tabular}


Tab. 10: (fortgesetzt)

\begin{tabular}{lllll}
\hline toponym & freq & sig & collocate & pos \\
\hline Sinai & 30 & 0.0001 & Gebäude & NN \\
Sinai & 36 & 0.0001 & bewaffnet & ADJA \\
Dominikanische Republik & 68 & 0.0001 & Republik & NN \\
Dominikanische Republik & 50 & 0.0001 & Dominikanische & NN \\
Katar & 63 & 0.0001 & Präsident & NN \\
Katar & 56 & 0.0001 & Todesfall & NN \\
Katar & 31 & 0.0001 & Fußball-Bund & NN \\
Katar & 49 & 0.0001 & Menschenrecht & NN \\
Katar & 22 & 0.0001 & Temperatur & NN \\
Katar & 50 & 0.0001 & Turnier & NN \\
Katar & 29 & 0.0001 & geplant & ADJA \\
Katar & 24 & 0.0001 & alarmierend & ADJA \\
Katar & 74 & 0.0001 & international & ADJA \\
Katar & 24 & 0.0001 & Situation & NN \\
\hline
\end{tabular}

Die Georeferenzierung von Toponymen ist dabei nicht trivial, und zwar aus folgenden Gründen:

- Ambiguität/Homonymität: Obwohl Eigennamen im Unterschied zu Gattungsnamen immer auf ein einzelnes Objekt referieren, sind sie nur im Kontext disambiguiert und ein Toponym wie „Berlin“ referiert potenziell auf mehrere Orte.

- Historizität: Toponyme wie „DDR“ oder „Sowjetunion“ waren nur zu bestimmten Zeiten offiziell anerkannte Ortsbezeichnungen. In einem aktuellen Ortsindex sind sie deshalb nicht mehr aufgeführt. Oder die Referenz hat seine Ausmaße verändert, wie etwa „Deutschland“ vor und nach dem 2. Weltkrieg.

- Strittigkeit: Toponyme wie „West-Sahara“, „Südossetien“, „Kosovo“ oder „Islamischer Staat“ sind keine international anerkannten Gebiete oder zumindest nicht als Staaten anerkannte Entitäten, die aber natürlich trotzdem benannt werden, wenn auch der genaue lokale Umfang der Referenz umstritten ist. 
- Orthographische und sprachliche Variation: Deutlich in historischer, aber auch in gegenwartssprachlicher Perspektive variiert die Schreibung von Toponymen („Milano“vs. „Mailand“, „Zürich Örlikon“vs. „Zürich Oerlikon“, „Pfannenstiel“ vs. „Pfannenstil“, „Lwiw“ vs. „Lwow“ vs. „Львів“, etc.) und es existieren inoffizielle, aber sehr gängige Bezeichnungen („Tschechei“ statt „Tschechien“, „Ex-DDR“ etc.) sowie abgekürzte Varianten („China“ statt „Volksrepublik China“, „Britannien“ oder „Großbritannien“ statt „Vereinigtes Königreich Großbritannien und Nordirland“).

Die „Geonames“-Ressource ${ }^{58}$, eine gemeinschaftlich erstellte Liste von Ortsbezeichnungen weltweit, nennt zu jeder toponymischen Entität eine breite Palette an Variation und führt teilweise auch historische Ortsbezeichnungen auf. Es kann also als Gazetteer-System verwendet werden, löst jedoch das Ambiguitätsproblem nicht. Dafür benutzten wir CLAVIN (Cartographic Location And Vicinity INdexer ${ }^{59}$ ), der aufgrund des im Kontext gefundenen weiteren Toponyme eine Dis-ambiguierung zu erreichen versucht. Dafür wird einerseits der Ort mit der höheren Einwohnerzahl gewählt, sowie Ortsreferenzen bevorzugt, die geografisch nahe beieinander liegen.

Im Ergebnis der Georeferenzierung liegen zu den Toponymen die jeweiligen geografischen Koordinaten vor, sowie die in Geonames verfügbaren Angaben wie Ortstyp (Staat, Stadt, Gemeinde etc.), Populationsgröße, Staatszugehörigkeit etc. Allerdings sind nur Punktreferenzen, keine Flächen verfügbar, was wir in einem weiteren Schritt zumindest für Staaten noch hinzugefügt haben.

\subsubsection{Vorläuferversionen und Genese}

Die Genese dieser Operationalisierung ist jedoch Ergebnis einer mehrjährigen Entwicklungsgeschichte. Ausgangspunkt waren meine Überlegungen vor dem Hintergrund eines ganz bestimmten Korpus, dem Text+Berg-Korpus (Bubenhofer et al. 2015b), einer Sammlung von alpinistischen Texten des „Jahrbuch des Schweizer Alpenclubs“ und der „Alpen“ von 1864 bis in die Gegenwart. In diesen Texten ist geografische Lokalisierung allgegenwärtig, allerdings meistens in viel lokalerer Ausprägung als bei der oben skizzierten Forschungsfrage nach diskursiv geprägten Weltsichten. Aber auch da war die Frage nach dem Sprechen über Landschaft Motivator, eine korpuslinguistische Antwort darauf zu finden. Um

58 Vgl. www.geonames.org (letzter Zugriff: 22. 9. 2020).

59 Vgl. https://github.com/Berico-Technologies/CLAVIN/ (letzter Zugriff: 22. 9. 2020). 
dies auszuprobieren, begann ich ein Script in der Programmiersprache Perl zu verfassen. Eigentlich würde man sich zunächst ein klares Konzept der zu lösenden programmiertechnischen Aufgabe erstellen, bevor die Programmierung beginnt. Scriptsprachen sind jedoch dazu geeignet, mit dem Gestus eines Lesers an Texte heranzugehen und die selben Operationen, die man als Leser mental oder mit Hilfe von Papier und Stift oder Exceltabelle vornehmen würde, maschinell durchzuführen - und das sofort ohne größere Planung (vgl. dazu Abschnitt 4.4.2). Ich habe mir jedoch angewöhnt, zu Beginn eines Scripts dessen geplante Aufgabe in zwei, drei Sätzen zu notieren. In Tabelle 11 (s.u., S. 216) ist der Kopf des Scripts, das ich „geoLinguistics“ taufte, aufgeführt. ${ }^{60}$

Das Script funktioniert zunächst also zur Erstellung eines einfachen Index, bei dem Toponymen Wörter zugeordnet werden, die im selben Satz erscheinen und diese zusätzlich gezählt werden. Ausgegeben wird dann eine XML-Datei dieses Indexes, die anschließend über eine HTML-Datei auf einer Karte visualisiert wird. Diese Visualisierung wiederum ist eine angepasste Version einer bereits in Bubenhofer (2009, 276-277) verwendeten Visualisierung ähnlicher Daten, bei der ich die Anzahl von Nennungen von „Staaten, Hauptstädten und Einwohner/ innen im NZZ-Korpus“ in Form von Datenpunkten auf Karten darstellte. Anstelle von Datenpunkten werden jedoch direkt die zu einem Toponym gehörenden Wörter am entsprechenden Ort dargestellt (vgl. Abbildung 68, S. 217).

Die Darstellung verwendet „Google Maps“ und die entsprechende API als Grundlage und ist mit Javascript realisiert. Diese Visualisierung ist offensichtlich nicht dafür gedacht, gezeigt zu werden, ist doch z. B. das Fenster mit Codes zum Debugging des Scripts dargestellt und befriedigt die Visualisierung generell nicht. Diese Vorarbeiten waren jedoch wichtige experimentelle Schritte hin zu einem verfeinerten Konzept von „Geokollokationen“, wie ich im Folgenden zeigen werde. Die Vorarbeiten veranschaulichen zudem den Einfluss technischer Umgebungen auf die Experimentierpraxis: Das wenige Zeilen enthaltende Perl-Script zur Berechnung der Wortfrequenzen zeigt noch Spuren des Herantastens an bessere Lösungen; mit der Zeit umfasste es über 600 Zeilen Code, der in diesem Zustand jedoch fern eines systematisch strukturierten Scripts ist, sondern auskommentierte Codeteile, Testzeilen und unsystematische Zwischenkommen-

60 Bemerkenswert ist, dass in der Programmierung von „lesen“ bzw. „read“ (und „schreiben“ - „write“) von Daten die Rede ist, auch wenn es sich nicht um Textdaten handelt. Auch in meiner Beschreibung ist mit „liest mit Geo-Informationen annotierte Textdateien...“ von Lesen die Rede und es wird deutlich geschildert, was pro Satz, der gelesen wird, getan werden soll. Dies zeigt einmal mehr, wie wichtig Text und das Schreiben (und Lesen) von Daten in der Konzeptualisierung des Computers ist, wie ich bereits in Abschnitt 4.2 und den Ausführungen zum Computer als Schreibmaschine erläuterte. 
Tab. 11: Ausschnitt Perlscript „geoLinguistics.pl“: Kopf der Datei mit Aufgabenstellung und Versionsänderungen

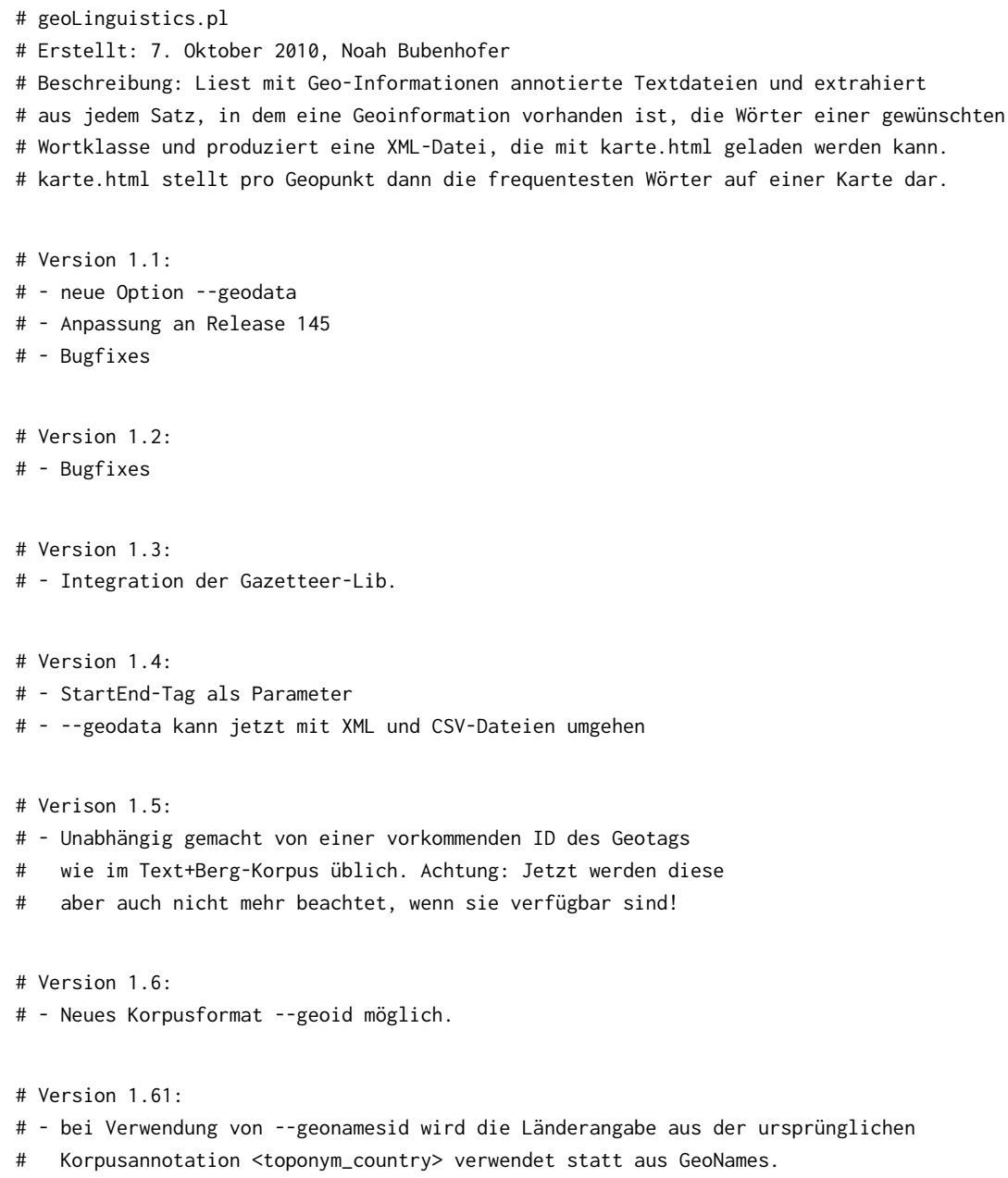

tare enthält. Scriptsprachen und vor allem die damit verbundene Coding Cultures, die Experimentierfreudigkeit begünstigen (vgl. Abschnitt 4.4.2), lassen ein solches Vorgehen zu.

Die Visualisierung basiert zudem auf Ideen und Code einer älteren Anwendung. Die dafür nötige HTML-Datei und der JavaScript-Code können direkt angepasst werden, um schnell erste Visualisierungsideen auszuprobieren. Der JavaScript-Code ist zudem durchsetzt mit Codeschnipseln von fremden Scrip- 


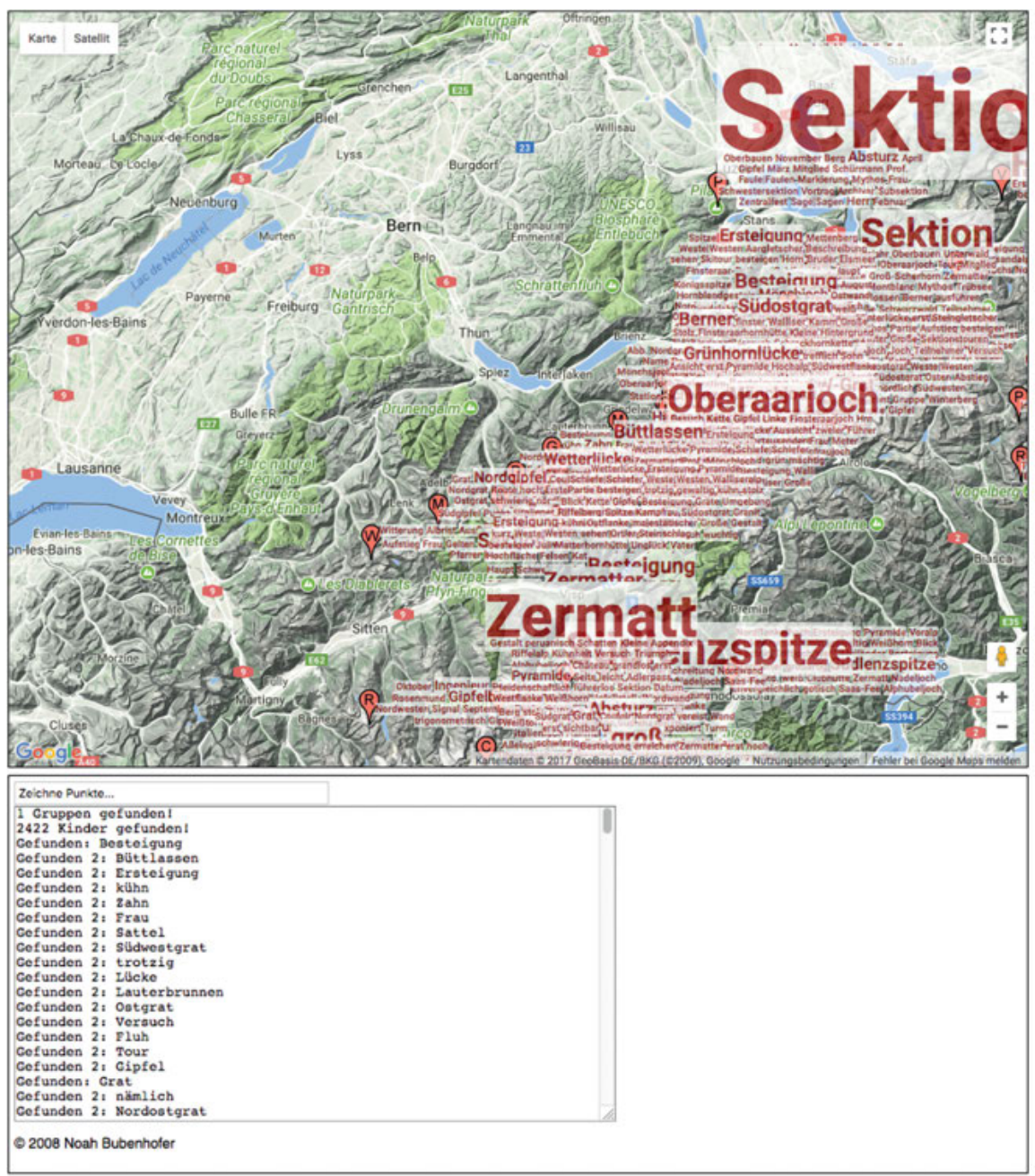

Abb. 68: Vorversion einer Darstellung von Wörtern in der Umgebung von Toponymen

ten, die von im Web verfügbaren Tutorials oder Beispielscripten kopiert worden sind. Das gesamte Script stellt also eine Collage von altem, neu angepasstem und fremdem Code dar. Mit der Verwendung der Google-API für die Kartendarstellung schreibt man sich zudem in die Coding Culture der Webservices ein, bei der die am Ende sichtbare Visualisierung eine Mischung von selbst- und fremdbestimmten Elementen darstellt. So kann die grafische Darstellung der Karte beispielsweise nur minimal beeinflusst werden und man ist abhängig von den Vorgaben der Firma Google und deren kommerziellen Interessen. 


\subsection{Visualisierung}

\subsubsection{Vorüberlegungen}

Wie oben in Abschnitt 6.1.1 beschrieben, sind bei der Berechnung von Geokollokationen mehrere diagrammatische Transformationen beteiligt: Vom Text zur Liste, zum Vektorraum und zur Karte. Alle diese Repräsentationsformen haben das Potenzial, auch grafisch visualisiert zu werden. Insbesondere könnten die Positionen der Toponyme zueinander im Vektorraum in einem auf zwei oder drei Dimensionen reduzierten grafischen Raum visualisiert werden: Die Toponyme können durch die Berechnung der Kollokationsprofile als Vektor repräsentiert werden. Die Vektoren von Toponymen, die in ähnlichen Kontexten verwendet werden, ähneln sich dann insofern, als dass die geometrische Distanz zwischen ihnen klein ist. Abbildung 69 zeigt ein Dendrogramm einer solchen Clusterberechnung, mit der die Ähnlichkeit von Toponymen aufgrund ihrer Kollokationsprofile berechnet worden sind. Die Toponyme ähneln den anderen Toponymen je nach Position im Dendrogramm. So bilden etwa die Länder USA, Japan, Indien, Russland, Amerika, Frankreich, China, Israel und Afghanistan ein Cluster auf der einen Seite des Baumes (in der Abbildung oben, vergrössert), einige Bundesländer Deutschlands sowie Karlsruhe ein anderes (in der Abbildung unten). Das Diagramm zeigt, dass Elemente einer diskursiv konstruierten Welt auch gänzlich ohne geografische Kartendarstellungen erfolgen kann und sich dabei gerade die Chance ergibt, die Ähnlichkeit der Toponyme losgelöst von ihrer geografischen Verortung zu interpretieren.

Die oben (Abschnitt 6.1.4) geschilderte Genese der Idee der Geokollokationen zeigt aber deutlich, dass die Repräsentation der Geokollokatoren im geografischen Raum von Beginn an dominant war. Nicht zuletzt liegt das auch daran, dass bereits Code zur Darstellung von Datenpunkten auf einer Google Maps-Karte zur Verfügung stand. Die darauf basierende experimentelle Darstellung der Geokollokatoren befriedigte jedoch nicht (vgl. Abbildung 68), vor allem deswegen, weil die Parametrisierung der über die Google API erstellten Karte zu wenig frei ist und die Darstellung nur beschränkt den eigenen Vorstellungen entsprechend angepasst werden kann. Deshalb musste das enge Korsett der technischen Möglichkeiten gesprengt werden.

Die Wünsche an eine verbesserte Darstellung waren demnach:

1. Die Geokollokationen als Ausdruck einer diskursiven Prägung von Welt sollen als eigene kartografisch darstellbare Informationsebene aufgefasst werden. Dies ergibt sich aus der Idee, dass die diskursiv geprägte Weltsicht ein Analogon zur geografischen Ebene darstellt und sozusagen strukturell an sie gekoppelt ist. Damit muss sich diese Ebene der gleichen diagramma- 


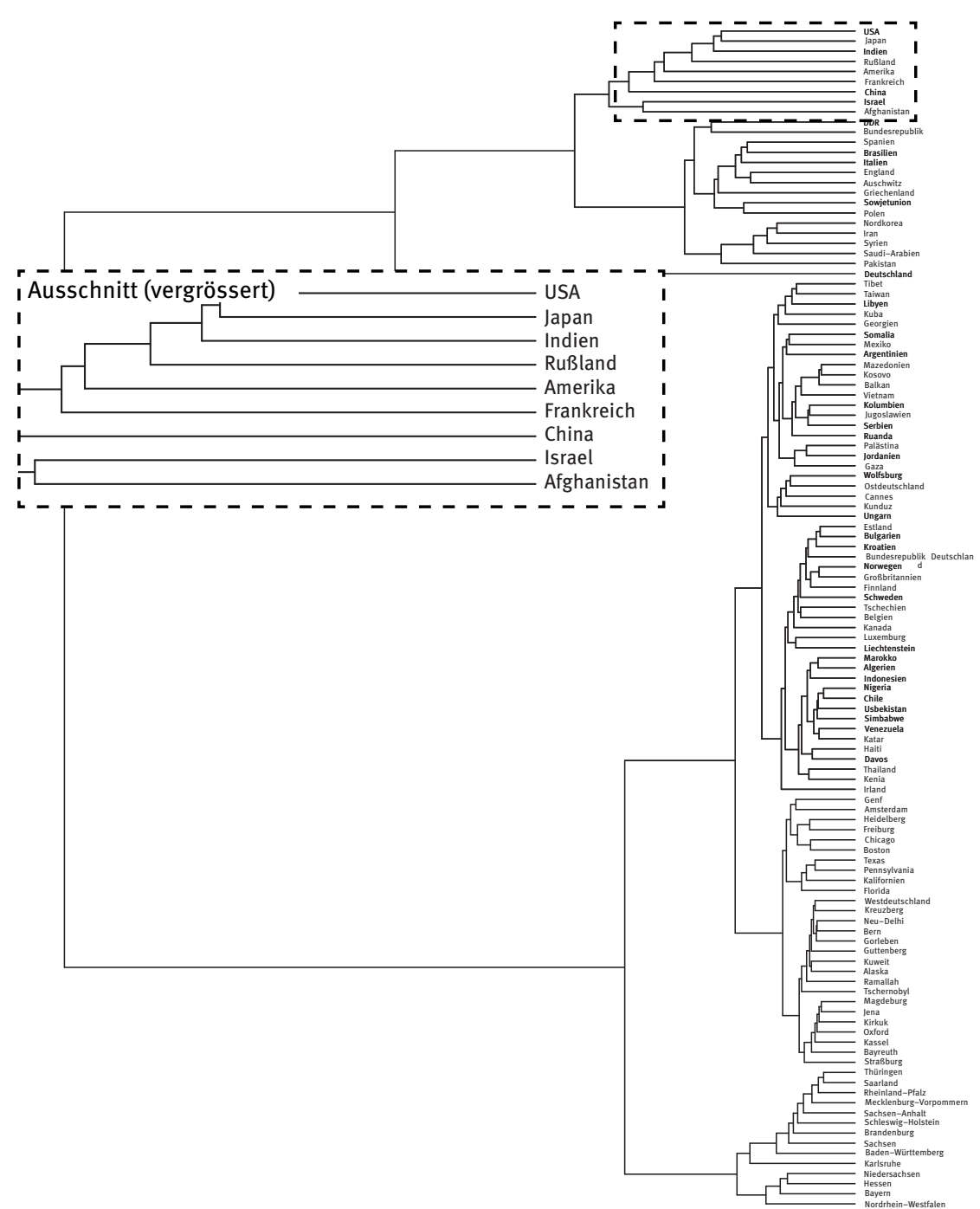

Abb. 69: Dendrogramm einer Clusterberechnung von Toponymen aufgrund ihrer Kollokationsprofile: Toponyme, die im Dendrogramm dem gleichen Zweig angehören, haben ähnliche Kollokationsprofile (Datengrundlage: Korpus Zeit/Spiegel 2010-2016, Kollokatoren mit $\mathrm{p}<=$ 0.0001 und Mindestfrequenz 11, 15'600 Kollokatoren, Clustering ward, euklidische Distanz)

tischen Grundfigur bedienen wie die Karte selbst, das bedeutet, sich dem Koordinatensystem der Karte, also deren Gerichtetheit sowie deren Relationalität und Referenzialität (vgl. Abschnitt 2.2) unterzuordnen. 
2. Da die Kollokationsprofile bereits eine mehrstufige Transformation von Text darstellt und als Repräsentationselement von Diskurs aufgefasst werden soll, soll die Visualisierung zunächst auf weitere Transformationen verzichten und die Geokollokatoren direkt in Textform anzeigen. Denkbar wäre ja, nicht Text, sondern die Kollokationsprofile und die damit verbundenen Vektoren abstrakt auf der Karte darzustellen. Dies könnte beispielsweise dadurch geschehen, dass die Position der Vektoren im Vektorraum in einem farblichen Kontinuum codiert ist und dann die Toponyme auf der Karte entsprechend eingefärbt würden; ähnlich eingefärbte Toponyme würden dann Nähe im Vektorraum und damit ähnliche semantische Prägung der Toponyme bedeuten. Damit ginge aber ein direkter Zugriff auf die Inhalte der Vektoren verloren. Wir versuchten aber im letzten Schritt der Visualisierungsexperimente diese Sicht auch noch zu integrieren (dazu vgl. Abschnitt 6.2.3).

3. Die interne Ordnung des Kollokationsprofils soll grafisch codiert werden: Als interne Ordnungsmöglichkeiten können absolute oder relative Frequenz des Kollokators dienen, mit der er zusammen mit dem Toponym erscheint oder aber das Assoziationsmaß der Kollokation. Natürlich sind weitere Ordnungskriterien denkbar wie alphabetische Ordnung, Ordnung nach Wortartklassen etc. oder Kombinationen davon.

4. Die Visualisierung soll verschiedene Filtermöglichkeiten zulassen, um den Datensatz in unterschiedlichem Umfang darzustellen.

5. Der Nachteil der direkten Anzeige der Geokollokatoren als Text (vgl. Punkt 2 oben) liegt natürlich darin, dass Ähnlichkeiten zwischen den Kollokationsprofilen nicht auf den ersten Blick sichtbar sind. Dabei ist aber schon auf Wortebene schnell sichtbar, dass bestimmte Kollokatoren bei vielen Profilen erscheinen, also sozusagen „global“ verwendet werden, andere sich auf einen oder wenige Profile beschränken und damit „lokal“, also spezifisch für bestimmte Orte, sind. Diese Verbreitungstypen der Kollokatoren soll darstellbar sein.

Die technischen Beschränkungen der bisherigen Lösung machten es somit notwendig, andere Lösungen zu finden, die ich im Folgenden zeige.

\subsubsection{Statische Visualisierungen}

Die im vorherigen Abschnitt genannten Vorüberlegungen und Bedingungen für die Visualisierung der Daten suggerieren ein strukturiertes Vorgehen: Planung gefolgt von Implementierung. Dies entspricht aber nicht der Realität. Stattdessen bestanden die ersten Schritte darin, die Möglichkeiten der Programmiersprache 
R für die Darstellung von Karten zu evaluieren und damit zu experimentieren. Die Wahl fiel auf R aufgrund der ausgebauten Möglichkeiten der Datenmanipulation und statistischen Analyse, gepaart mit grafischer Ausgabe.

Als Grundlage für die Kartendarstellung in $\mathrm{R}$ diente das „World Borders Dataset"61, das die Umrisse aller Länder der Welt als Polygone enthält. Mit dem Paket „,rgdal“62 von Roger Bivand et al. kann dieses Dataset verarbeitet und dargestellt werden. Um die Geokollokationen darzustellen, wurde die Liste als Wortwolke aufgefasst und das Paket „,wordcloud“63 von Ian Fellows verwendet. Damit folgt die Anordnung der Kollokatoren einer eigenen diagrammatischen Logik, die das Ziel verfolgt, die Wörter nicht überlappend auf der Fläche zu verteilen, wobei Größe und Farbe jedes Wortes festgelegt werden können. Die Wortwolke wird an der Position des georeferenzierten Toponyms auf der Karte platziert. Abbildungen 70 und 71 zeigen die so produzierten Karten, allerdings bereits mit weiteren grafischen Elementen.

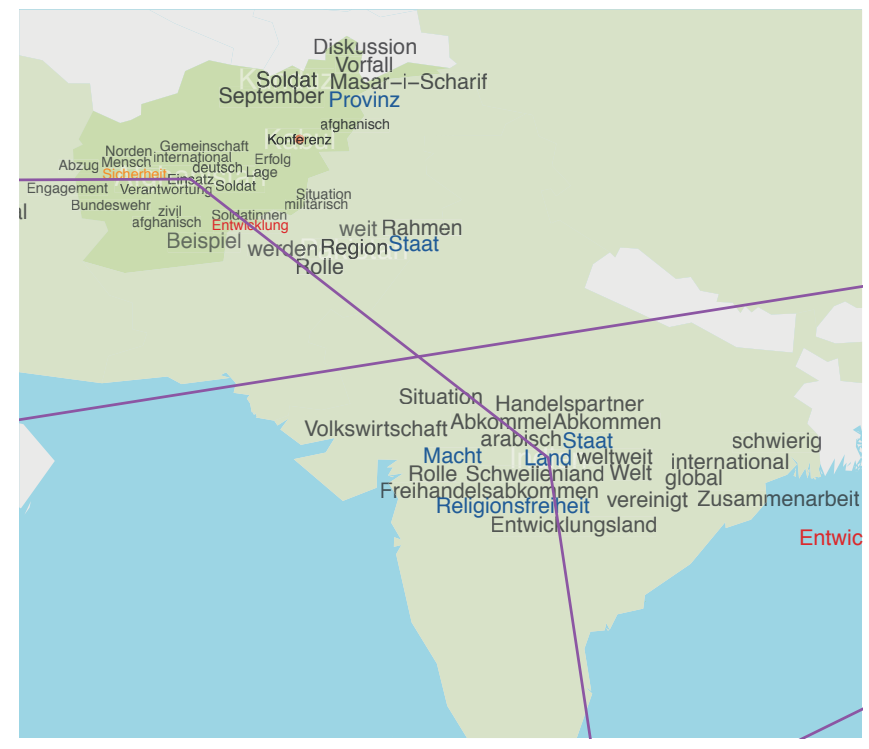

Abb. 70: Ausschnitt aus einer mit R produzierten Karte von Geokollokationen (Korpus: Bundestag WP 17, CDU/CSU-Fraktion)

61 Vgl. http://thematicmapping.org/downloads/world_borders.php (letzter Zugriff: 22. 9. 2020). 62 Vgl. https://cran.r-project.org/package=rgdal (letzter Zugriff: 22. 9. 2020).

63 Vgl. https://cran.r-project.org/web/packages/wordcloud/wordcloud.pdf (letzter Zugriff: 22.9.2020). 
Wie bereits zu den Coding Cultures ausgeführt (vgl. Abschnitt 4.4.2), ist auch $\mathrm{R}$ dadurch gekennzeichnet, ein Arbeitsinstrument zu sein, wo Programmierung und Anwendung ineinander greifen. Auch das gut 400 Zeilen umfassende R-Script ist geprägt von auskommentierten Zeilen und Kommentaren, die vom Experimentieren mit verschiedenen Varianten zeugen. Die beiden Abbildungen 70 und 71 zeigen Varianten, bei denen Kollokatoren bestimmter semantischer Klassen farblich markiert sind. Für sie semantische Klassifizierung wurden Dornseiff-Kategorien verwendet (Dornseiff 2004), also Sachgruppenbezeichnungen wie „Krieg“, „Streit“, „Kampf“ etc. (rot) oder „Politik“, „Regierung“, „Wahl“ etc. (blau). Über einen Abgleich mit den Sachgruppen wurden die Kollokatoren automatisch zugeordnet, wobei Kollokatoren mehreren Sachgruppen angehören können.

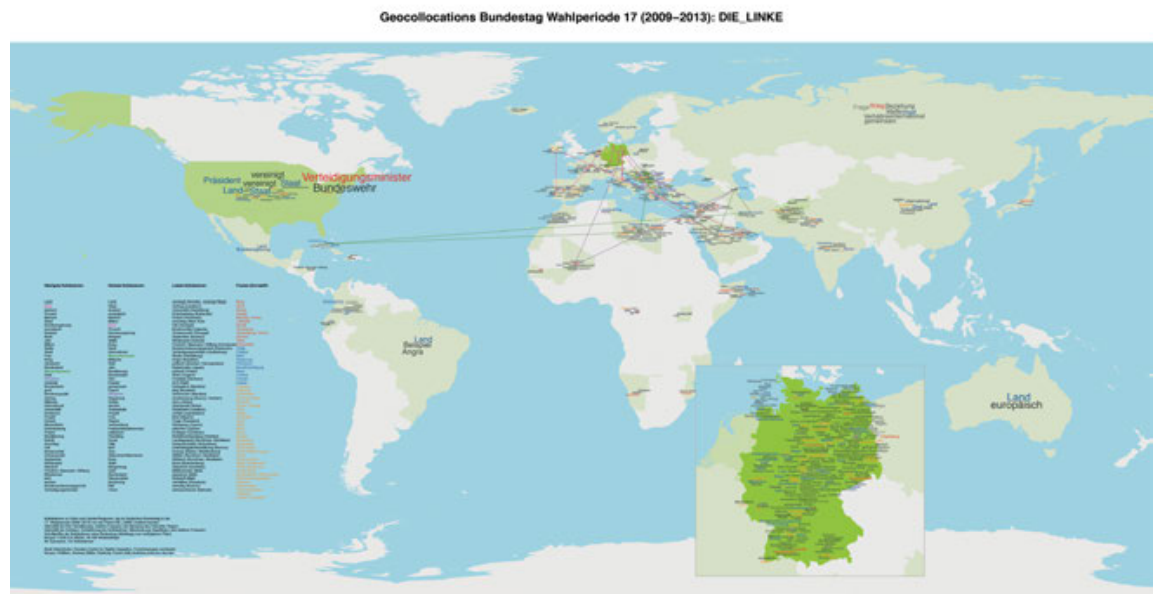

Abb. 71: Vollansicht einer mit R produzierten Karte mit Geokollokationen (Korpus: Bundestag WP 17, Fraktion Die Linke)

Eine andere Weiterentwicklung betrifft das Berechnen von „globalen“ und „lokalen“ Kollokatoren. Globale Kollokatoren sind solche, die über weite Teile der Erde streuen, lokale, die nur an einem bestimmten Ort verwendet werden. Um ein Maß für die Globalität und Lokalität zu erhalten, wurden die Koordinaten der jeweiligen zum Kollokator gehörenden georeferenzierten Toponyme in aufsteigender Reihenfolge geordnet und dann die euklidischen Distanzen dazwischen aufsummiert. Je größer die euklidische Distanz, desto globaler wird der Kollokator verwendet. 
In Abbildung 71 sind auf der linken Seite Listen der globalsten und lokalsten Kollokatoren sichtbar. Da die Listen in der Abbildung wegen der Verkleinerung nicht lesbar sind, seien hier die jeweils ersten zwölf genannt:

- Globale Kollokatoren: Land, Staat, deutsch, europäisch, Mensch, Million, Euro, Prozent, Bundesregierung, Beispiel, Waffe, Krieg

- Lokale Kollokatoren (in Klammer häufigstes assoziiertes Toponym): vereinigt (USA), Vertrag (Lissabon) ${ }^{64}$, Universität (Heidelberg), Entscheidung (Karlsruhe) ${ }^{65}$, Putsch (Honduras), Anschlag (New York), Fall (Senegal), Kindersoldat (Uganda), Schwerpunkt (Senegal), September (Kunduz) ${ }^{66}$, Winterspiel (Sotschi), Friedrich-Naumann-Stiftung (Honduras) ${ }^{67}$

Im R-Code können bestimmte Kollokator-Types bestimmt werden, die bei der Visualisierung mit Kanten verbunden werden, um ihre globale Verbreitung zu sehen.

Eine Schwierigkeit der so entstandenen Darstellung ist die ungleiche Verteilung der Kollokatoren über die Welt. Je nach Korpus kommt es zu Schwerpunkten; so werden in den meisten Korpora deutschsprachiger Texte (Zeitungen, Bundestagsprotokolle etc.) häufiger Toponyme in Europa und besonders Deutschland genannt, was zu unleserlichen Darstellungen in diesen Regionen führt. Deshalb kann im R-Code ein geografischer Bereich definiert werden, der aus der Übersichtsdarstellung ausgesondert und vergrößert dargestellt werden soll.

Solche Probleme verdeutlichen natürlich die Schwierigkeiten einer statischen Darstellung. Die mit dem R-Code entstehenden Visualisierungen, im SVGoder PDF-Format abgespeichert, werden sehr groß und unhandlich. Für eine damals entstandene Publikation (Bubenhofer 2014) erstellte ich als Ergänzung eine Website, die eine größere Auswahl an Darstellungen, zudem in Farbe, aufführte, die im Zeitschriftenartikel nicht abgedruckt werden konnten. ${ }^{68}$

64 Zurückgehend auf den „Vertrag von Lissabon zur Änderung des Vertrags über die Europäische Union und des Vertrags zur Gründung der Europäischen Gemeinschaft“" von 2007, der 2009 in Kraft trat.

65 Zurückgehend auf Entscheidungen des Bundesverfassungsgerichts in Karlsruhe.

66 Diese Verbindung geht auf den am 4.11.2009 von einem deutschen Oberst angeforderten und US-Streitkräften ausgeführten Luftangriff auf zwei Tanklastwagen zurück, bei dem eine große Zahl ziviler Opfer entstanden und was zu politischen Debatten in Deutschland führte.

67 Diese Verbindung geht darauf zurück, dass 2009 die Stiftung Verständnis für einen Putsch in Honduras zeigte; vgl. Tagesspiegel (14.8.2009): „Friedrich-Naumann-Stiftung rechtfertigt Putsch in Honduras“; http://www.tagesspiegel.de/politik/staatsstreich-friedrich-naumann-stiftung-rechtfertigt-putsch-in-honduras/1579132.html (letzter Zugriff: 22. 9. 2020).

68 Vgl. http://www.bubenhofer.com/geocollocations/ (letzter Zugriff: 22. 9. 2020). 
Das Ziel der dynamischeren Visualisierung sollte sein, die Interaktion mit der Karte $\mathrm{zu}$ vereinfachen. Im Prinzip ist auch die sogenannt „statische“ Version hochgradig interaktiv, da mit den verschiedenen R-Code-Schritten, die ausgelöst werden, die Datenaufbereitung und Darstellung weitgehend beeinflusst werden kann. Denn die Visualisierung ist ja streng genommen nicht bloß die in Abbildung 71 gezeigte Karte, sondern die Kombination aus R-Script und Karte, wobei für die Arbeit mit einem R-Script oft eine Softwareumgebung wie „RStudio“69 verwendet wird, die als Arbeitsbank angesehen werden kann und die Programmierung und Analyse vereint (vgl. Abbildung 72). Die Arbeit mit einer solchen Umgebung hat den Vorteil, dass sie eine White Box darstellt, bei der (im Prinzip, wenn der Code verstanden wird) alle Schritte transparent sind.

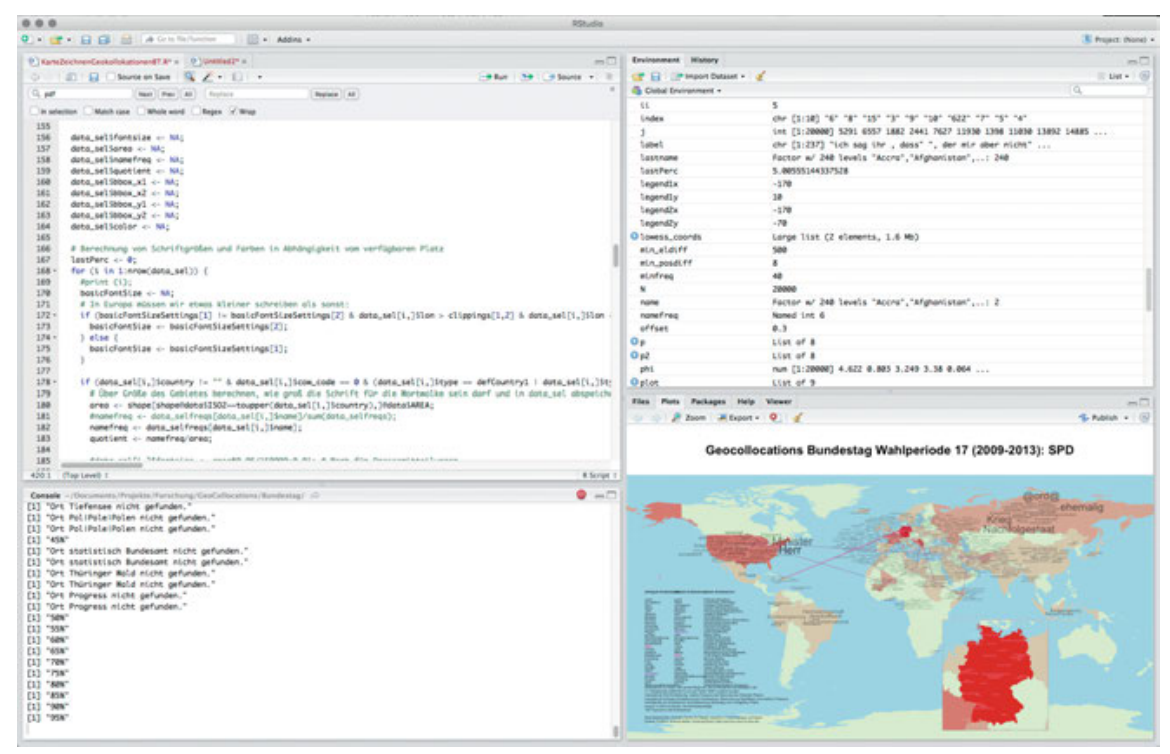

Abb. 72: Die Arbeit mit einem R-Script in R-Studio

Allerdings dauert ein Durchlauf durch das ganze Script pro Karte ungefähr eine Minute - egal welches Detail der Darstellung geändert wird. Daher ist der Wunsch nach einer Umgebung, mit der schneller mit der Visualisierung interagiert werden kann, sinnvoll und verständlich.

69 Vgl. https://www.rstudio.com/ (letzter Zugriff: 22.9.2020). 


\subsubsection{Dynamische Visualisierungen}

Es ist inzwischen problemlos möglich, mit $\mathrm{R}$ interaktive Web-Anwendungen zu erstellen, so etwa mit „Shiny“ von RStudio. Die Firma RStudio wurde 2008 gegründet, die Software RStudio 2011 erstmals veröffentlicht. ${ }^{70}$ Shiny wiederum muss Ende 2012 erschienen sein. ${ }^{71}$ Fast zwei Jahre vorher erschien die JavascriptBibliothek „D3.js“ erstmals (Bostock et al. 2011), die explizit dafür gemacht ist, interaktiv und dynamisch Daten im Webbrowser zu visualisieren. Mit $\mathrm{R}$ und Javascript/Webtechnologien stehen also auch zwei unterschiedliche Coding Cultures einander gegenüber, wobei sich $\mathrm{R}$ mit Entwicklungen wie Shiny an die Javascript/Webtechnologien-Kultur annähert (da Shiny ebenfalls Javascript benutzt und im Browser funktioniert). Um einen Eindruck der Popularität der beiden Technologien zu erhalten, sind in Abbildung 73 „Google Suchtrends“ von drei einschlägigen Suchabfragen aufgeführt. Man sieht dort, dass Suchanfragen nach „d3.js“ ab Mitte 2011 stark ansteigen und sich Anfang 2014 auf hohem Niveau stabilisieren. Anfragen nach „r shiny“ steigen erst ab Ende 2012 kontinuierlich an und überflügeln aber die Suchen nach „d3.js“ erst Anfang 2017. Damit einher geht eine Zunahme der Suchen nach „r programming“, also zu R generell. ${ }^{72}$

70 Vgl. https://www.rstudio.com/about/ (letzter Zugriff: 22. 9. 2020).

71 Es ist schwierig, das Geburtsdatum der Software zu bestimmen. Auf GitHub, der Plattform, wo der Code gelagert wird, datieren die ersten „Issue Requests“ von November 2012 (https:// github.com/rstudio/shiny/issues?q=is\%3Aissue+is\%3Aopen+sort\%3Acreated-asc). In der für Programmierprobleme beliebten Plattform „stackoverflow“ datiert die erste Frage dazu auch vom 9. November 2012 (https://stackoverflow.com/questions/13313503/r-studio-shiny-conditional-statements). In den offiziellen Release Notes von Shiny sind keine Daten angegeben (https:// shiny.rstudio.com/reference/shiny/0.11/upgrade.html). (Alle URLs zuletzt am 22.9.2020 geprüft.)

72 Relativierend muss jedoch betont werden, dass die Berechnungsmethode von Google hinter diesen Suchtrends unbekannt ist. Zum Maß der Trends, „Interessen im zeitlichen Verlauf“ genannt, schreibt Google: „Die Werte geben das Suchinteresse relativ zum höchsten Punkt im Diagramm für die ausgewählte Region im festgelegten Zeitraum an. Der Wert 100 steht für die höchste Beliebtheit dieses Suchbegriffs. Der Wert 50 bedeutet, dass der Begriff halb so beliebt war und der Wert 0 entspricht einer Beliebtheit von weniger als $1 \%$ im Vergleich zum Höchstwert“ (vgl. https://trends.google.de/, letzter Zugriff: 24.11.2017). Diese Erklärung ist ungenügend, um die Werte richtig interpretieren zu können, zumal sie auch nichts über das Verhältnis der Werte für die einzelnen Suchbegriffe zueinander aussagt. 


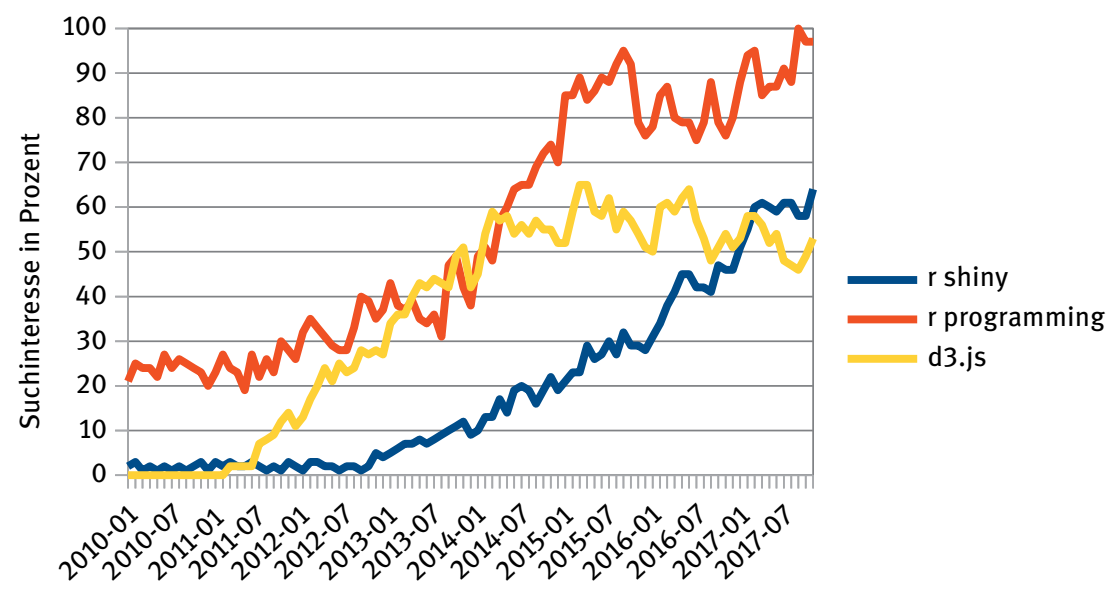

Abb. 73: Google Suchtrends für die angegebenen Suchbegriffe (vgl. https://trends.google.de); Suchinteresse in Prozent in Relation zum höchsten Wert

Dies bedeutet, dass noch 2015, als wir nach technischen Möglichkeiten einer interaktiveren Version der Geokollokationen suchten, D3.js wahrscheinlich die populärere Bibliothek war als Shiny. Heute, 2017, wäre der Schritt von der statischen R-Version zu einer interaktiveren mit Shiny oder einer ähnlichen Bibliothek ebenfalls naheliegend.

Ausgangspunkt für die erste D3.js-Version der Geokollokationen-Visualisierung waren einerseits die eigenen Vorarbeiten, andererseits verschiedene Beispiele für Kartenvisualisierungen mit D3 in der Community. Abbildung 74 zeigt eine Ansicht der Visualisierung.

Neu mit dieser Version gibt es als Ergänzung zur zoom- und verschiebbaren Kartendarstellung ein Bedienfeld, um die Darstellung zu beeinflussen. Folgende Auswahlmöglichkeiten stehen zur Verfügung:

- Auswahl der darzustellenden Wortklassen der Kollokatoren: Alle, Nomen, Adjektive, Verben, andere.

- Aggregation der Daten: Grundeinstellung nach Ort (jeder georeferenzierte Toponym-Type stellt einen eigenen Ort auf der Karte dar), Zusammenfassung nach Staaten (Kollokatoren zu Orten im selben Staat werden aggregiert).

- Dornseiff-Sachgruppen: Eine Liste nennt alle Sachgruppen, die durch Kollokatoren auf der Karte vertreten sind, inkl. der Anzahl der Vertreter. Ein Auswahl von Sachgruppen reduziert die Anzeige der Kollokatoren auf die jeweiligen Vertreter der Sachgruppen.

- Signifikanzniveau: Auswahl des Signifikanz-Schwellwerts der Assoziationsstärke, ab der die Kollokatoren angezeigt werden sollen. 


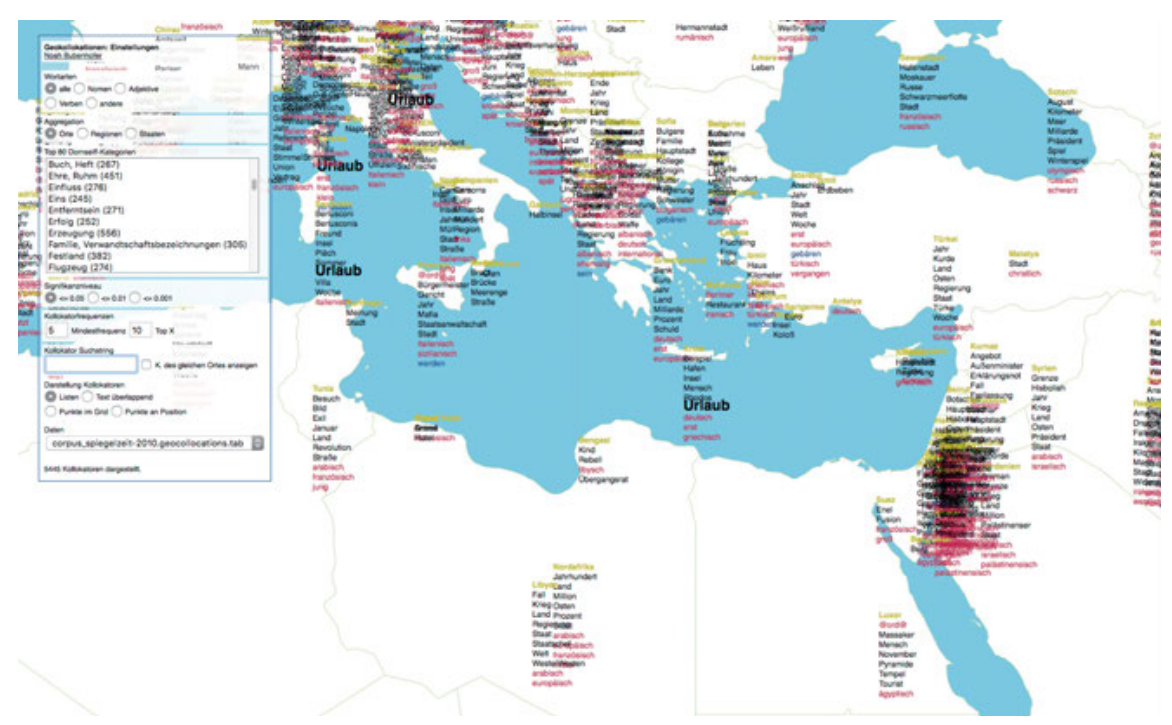

Abb. 74: Webinterface Geokollokationen Version 1: Übersicht

- Kollokatorfrequenzen: Auswahl der absoluten Mindestfrequenz der Geokollokation und Beschränkungsmöglichkeit auf die x häufigsten Kollokatoren pro Ort.

- Kollokator Suchzeichenkette: Auswahl von Kollokatoren, die auf einen Suchausdruck (Syntax „Reguläre Ausdrücke“) passen.

- Darstellungsarten der Kollokatoren: Alternative Darstellungsweisen der Kollokatoren (vgl. dazu die folgenden Ausführungen).

- Datenauswahl: Auswahl des Korpus.

Mit den verschiedenen Auswahlmöglichkeiten bewegt sich die Visualisierung stärker hin zu einem explorativen Werkzeug. Eine offene Frage blieb aber immer noch die Darstellung der Kollokatoren auf der Karte. Die Darstellung nach dem Muster von Wortwolken, wie in der statischen Visualisierung (vgl. Abschnitt 6.2.2) scheiterte aus technischen Gründen, da dafür keine einfache algorithmische Lösung gefunden werden konnte. ${ }^{73}$ Allerdings befriedigte sie auch nicht vollends, da die Position der Kollokatoren innerhalb der Wortwolke nicht semantisiert und damit der Vorteil gegenüber einer Liste unklar ist.

73 Selbstverständlich ist es mit Javascript und D3 möglich, eine solche Darstellung zu implementieren. Da ich jedoch in akzeptabler Frist keine Lösung fand, gab ich die Suche danach auf. 
Zudem stellt sich natürlich die Frage, ob die Kollokatoren überhaupt einzeln auf der Karte dargestellt werden sollen, zumal für bestimmte Anwendungen gerade eine abstrahierte Sicht auf die Daten relevanter ist: Nicht die einzelnen Kollokatoren sind dann interessant, sondern deren Zusammenfassung.

So implementierte ich vier verschiedene Darstellungsweisen der Kollokatoren in die Visualisierung, wovon Abbildung 75 zwei zeigt. Anstelle von Wortwolken werden Listen verwendet, die die Kollokatoren nach Wortartklasse (zusätzlich farbcodiert) und danach alphabetisch geordnet aufführten. Sie werden mit dem entsprechenden Toponym übertitelt. Geografischer Ankerpunkt der Listen ist die obere linke Ecke.
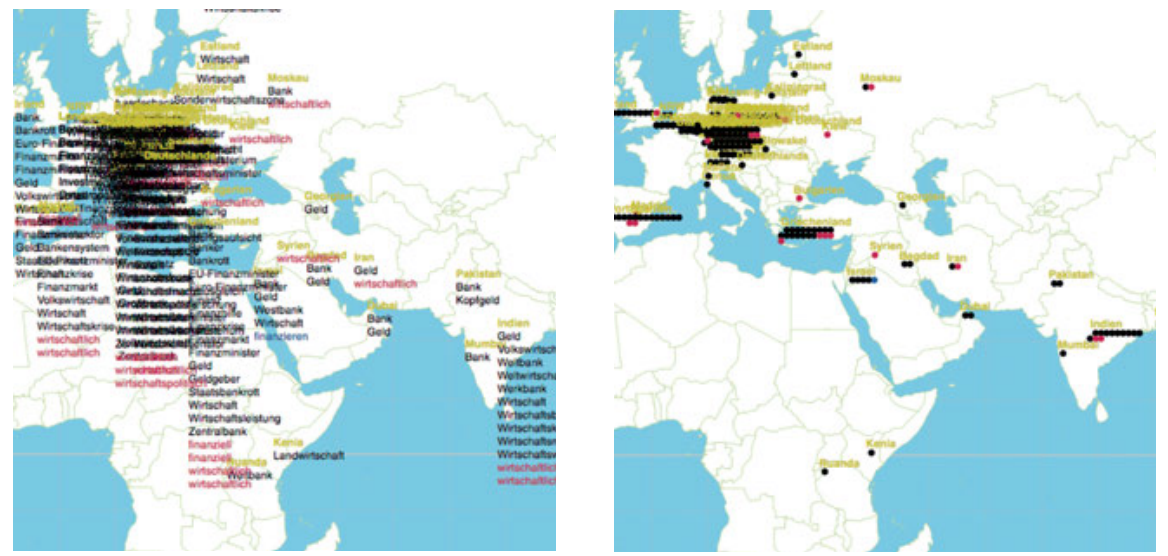

Abb. 75: Darstellung der Kollokatoren als Listen oder Punkte

Wie bereits Abbildung 76 zeigt, ist das Problem dieser Listen deren Positionierung in dichten Bereichen, wo es zu starken Überlappungen kommt und die Listen unleserlich werden. Sie zeigen in solchen Bereichen zwar noch Dichte an, erfüllen ihren Zweck jedoch nicht mehr vollständig. Als Alternative bietet sich eine Punktdarstellung an (vgl. Abbildung 75, rechte Seite), wobei jeder Punkt für einen Kollokator steht und sie in einem Raster von maximal zehn Punkten Breite und beliebiger Höhe angeordnet werden. Die Farbgebung codiert wiederum die Wortartklassen-Zugehörigkeit. Auch hier kann es zu Überlappungen kommen, jedoch nicht mehr zu so gravierenden, wie bei den Listen. 


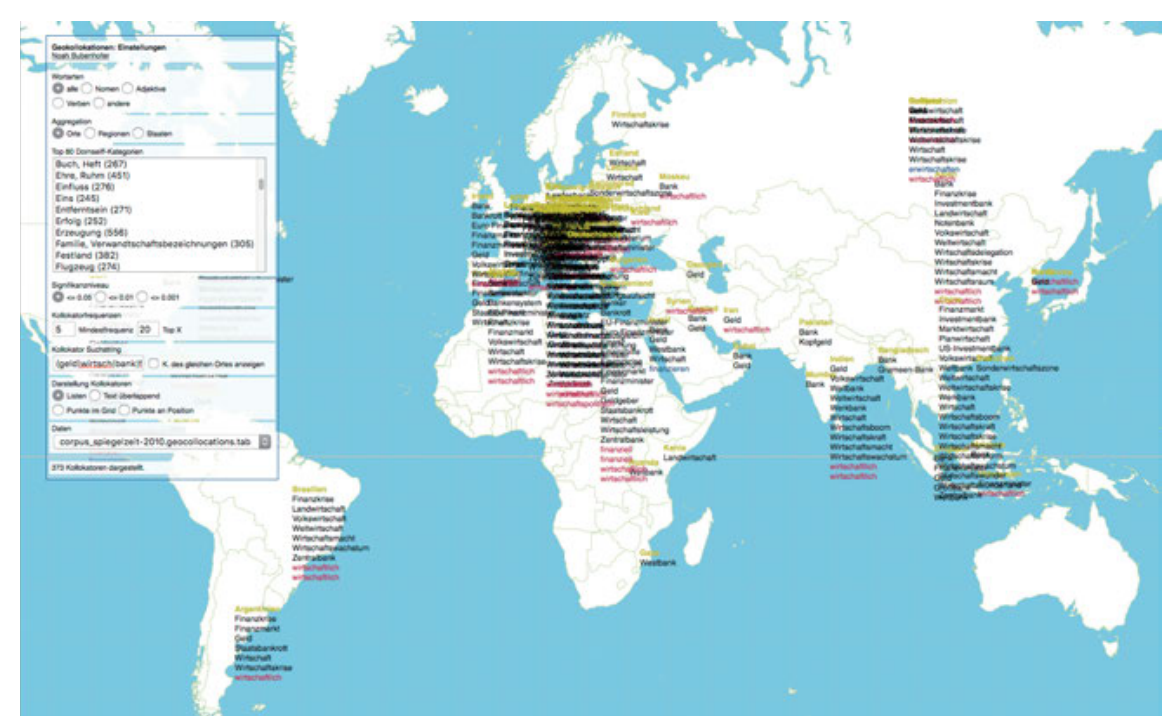

Abb. 76: Geokollokationen Version 1: Auswahl von Kollokatoren, die eine der folgenden Zeichenketten enthalten: geld, wirtschaft, bank, finanz

Je nach Forschungsinteresse interessieren bestimmte thematisch definierte Diskurse. Abbildung 76 zeigt ein Beispiel für die Beschränkung der Darstellung auf Kollokatoren, die einen Wirtschafts- und Finanzdiskurs repräsentieren sollen. Interessant bei einer solchen Auswahl ist nicht nur die geografische Verteilung und die Anzahl der Kollokatoren, sondern auch eine Analyse der Derivationen, z. B. von Komposita, etwa um sich für die weitere Analyse das relevante Wortfeld zu erschließen.

Schließlich zeigt Abbildung 77 ein Beispiel für die Auswahl bestimmter Dornseiff-Kategorien. Im Beispiel sind es die Sachgruppen „Angriff“, „Kampf“, „Kämpfer/Armee“, „Krieg“, „Streit“ und „Töten“ ausgewählt. Das Untersuchungsinteresse lag hier darin, Kriegsdiskurse zu untersuchen. Angezeigt werden damit nur Kollokatoren, die diesen Sachgruppen angehören. Damit kommen semantisch zusammenhängende Kollokatoren in den Blick. Allerdings müsste für eine sinnvolle und ergiebigere Analyse die Zuordnung zu den Sachgruppen verbessert werden, etwa indem die Lexeme im Korpus disambiguiert werden oder eine gänzlich andere Methode zur semantischen Annotation benutzt würde. Obwohl die Dornseiff-Kategorien sinnvoll für semantische Analysen eingesetzt werden können (vgl. dazu Scharloth et al. 2013; Bubenhofer et al. 2015a), hielt sich der Nutzen im Fall der Geokollokationen in Grenzen. Das liegt vor allem daran, dass einzelne Kollokatoren niemals eindeutig einer bestimmten Sachgruppe zugeord- 
net werden können, zumal ohne den weiteren Kontext für eine Disambiguierung zu nutzen. Deshalb wurden die Dornseiff-Kategorien für die weiteren Visualisierungsexperimente nicht mehr weiter benutzt.

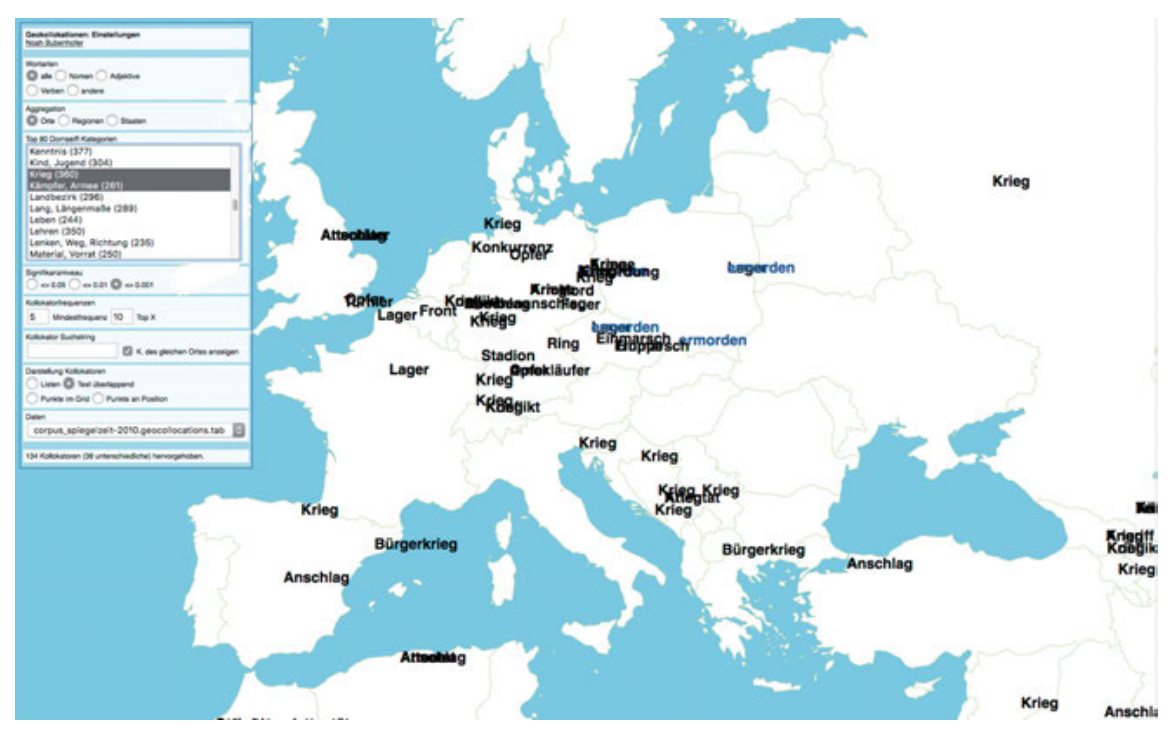

Abb. 77: Geokollokationen Version 1: Darstellung von Sachgruppen nach Dornseiff

\subsubsection{Erweiterte Version 2.0}

Eine Erweiterung des Teams um die Informatikstudentin Katrin Affolter, die Computerlinguistikstudentin und Spezialistin für Informationsvisualisierung Danica Pajovic und den Linguisten/Computerlinguisten Klaus Rothenhäusler führte zu weiteren Diskussionen zur Visualisierung der Geokollokationen. Die drei lenkten den Fokus auf die mangelhafte programmiertechnische Implementierung und die Entwicklung weiterer Visualisierungsideen. Dabei ist Folgendes interessant: Nur ich selbst benutzte das Geokollokationen-Werkzeug, um tatsächlich zumindest in Ansätzen diskurslinguistische Studien durchzuführen. Je mehr Leute an der Entwicklung beteiligt sind, desto stärker ergeben sich personelle Spezialisierungen zwischen Anwendung, visueller Konzeption, Design und Programmierung. Damit steigt der Koordinationsaufwand an, um eine gemeinsame Verständigung über die Ziele und Wege der Arbeit zu erhalten, und es wird besonders wichtig, das Praxiswissen im Umgang mit der Anwendung zu teilen. Dies erreichten wir nur zu 
Teilen, so dass sich die Anwendung zeitweise auch in Richtungen entwickelte, die für diskurslinguistische Untersuchungsinteressen nicht dienlich waren.

Die neuen Ideen führten $\mathrm{zu}$ teilweise erneuertem Code und einer anderen Datenbankanbindung: Statt in einer relational organisierten PostgreSQL-Datenbank $^{74}$ werden die Daten seither in einem Elasticsearch-Index ${ }^{75}$ vorgehalten. Hinter Postgres und Elasticsearch stehen nicht bloß zwei verschiedene Datenbankprogramme, sondern zwei unterschiedliche Prinzipien der Datenorganisation, nämlich sogenannte SQL vs. NoSQL-Prinzipien. ${ }^{76}$ Relationale Datenbanken wie PostgreSQL verlangen ein vorher definiertes Datenbankschema, das normalerweise dem Prinzip folgt, Daten in verschiedenen Tabellen abzulegen, die miteinander verknüpft werden. Im Beispiel der Geokollokationen existieren die drei Tabellen „lexemes“, „places“ und „collocations“. In „lexemes“ sind alle vorkommenden Lexeme aufgeführt, in „places“ alle Orte (mit den Angaben zur Georeferenzierung) und „collocations“ verknüpft die beiden Tabellen insofern miteinander, als dass es für jede Kollokation einen Eintrag dort gibt, der besagt, welche Lexem-ID mit welcher Place-ID verknüpft ist. Dazu können dann weitere Informationen zur Frequenz, Signifikanz etc. angegeben werden.

Anders das auf dem Datenbanksystem „Lucene“"77 basierende Elasticsearch, welches dokumentenzentriert arbeitet und für schnelle Volltextsuchen in großen Datenmengen optimiert ist. Jedes darin befindliche Dokument kann eine eigene Struktur haben und es gibt kein datenbankweites gemeinsames Schema. Das System sorgt selbst für eine optimale Indizierung und ggf. Aufteilung der Daten und der Prozessierung der Suchabfragen auf verschiedene Server. Im Fall der Geokollokationen sind die Kollokationsprofile zu den Toponymen die „Dokumente“. Sie enthalten gleichzeitig auch die Georeferenzen und weitere Angaben zu Frequenz und Signifikanz, die in der relationalen Datenbank auf andere Tabellen ausgelagert sind.

Die Entscheidung, ob eine relationale SQL oder eine NoSQL-Datenbank verwendet wird, führt also zu unterschiedlichen Ansätzen der Datenmodellierung und ermöglicht unterschiedliche diagrammatische Operationen der Datenmanipulation. Oder konkreter: NoSQL-Datenbankstrukturen bedingen ein anderes diagrammatisches Denkmodell als SQL-Datenbanken.

74 Vgl. https://www.postgresql.org (letzter Zugriff: 22.9.2020).

75 Vgl. https://www.elastic.co/ (letzter Zugriff: 22. 9. 2020).

76 „SQL“ steht für „Structured Query Language“ und meint die für die Erstellung, Verwaltung und das Abfragen von relationalen Datenbanken nötige Datenbanksprache. „NoSQL“ bedeutet eben „not SQL“ oder „not only SQL“ und steht für nicht-relationale Datenbanksysteme.

77 Vgl. https://lucene.apache.org/core/ (letzter Zugriff: 22. 9. 2020). 
Die Organisation des Javascript-Codes wurde ebenfalls optimiert, indem er modularisiert, also auf verschiedene Dateien aufgeteilt wurde (Affolter 2016a). Die grundlegende Technologie veränderte sich ansonsten aber gegenüber der Vorversion nicht. In Kombination mit der neuen Datenbankanbindung veränderte sich aber die Aufgabenverteilung zwischen Server und Client. Der Client, also das Browserprogramm auf dem Computer der Nutzerin/des Nutzers, der den Javascript-Code interpretiert und die Darstellung erzeugt, übernimmt in der neuen Version weniger Aufgaben der Datenselektion. In der Version davor lieferte der Server den gesamten Datensatz an den Client und die Auswahl bestimmter Datensätze (bestimmter Toponyme, Anzeige bestimmter Kollokatoren) geschah mittels Javascript-Code beim Client. In der neuen Version geschieht die Selektion auf dem Server und dieser liefert nur die tatsächlich für die Darstellung relevanten Daten. Es gibt selbstverständlich ein technologisches Argument dafür, mehr Aufgaben dem Server zu überlassen, nämlich Geschwindigkeit: Der Server ist bezüglich Hardware normalerweise besser ausgestattet als die Clients und muss immer wieder gleiche Operationen nicht immer neu ausführen, sondern kann die Ergebnisse zwischenspeichern und so schneller ausgeben. Doch das Aufteilungsprinzip zwischen Client und Server gestaltet sich eben auch vor dem Hintergrund von Coding Cultures mit Auswirkungen auf das Verhältnis zwischen Mensch, eigenem Computer und Servern, insbesondere was die Kontrolle über die Daten und damit auch die Analysemöglichkeiten betrifft. In unserem Setting ist dieser Aspekt selbstverständlich vernachlässigbar, da wir kompletten Zugriff auf den Server haben und wir das Interface so gestalteten, dass der komplette Datensatz ohne jegliche Selektion immer abgerufen werden kann. Aber bei der Weitergabe der Anwendung für Nutzerinnen und Nutzer außerhalb des Entwicklerkreises ohne Zugriff auf den Server erhöht man damit die Intransparenz über die Datenstruktur und -Manipulation. ${ }^{78}$ In Abbildung 78 ist beispielhaft die Ausgabe der Variable „data“ während der Nutzung der Geokollokationen-Anwendung abgebildet, die nicht nur die Daten selbst, sondern auch deren Strukturierung preis gibt. Nur ein Teil dieser Angaben wird in der Visualisierung überhaupt verwendet. Da aber diese Informationen für jeden Nutzer/jede Nutzerin einsehbar ist, tragen alle clientseitige Operationen immer zu Transparenz der Anwendung bei.

78 In „normalen“ Nutzungskontexten ist vielleicht gar nicht deutlich, wie transparent clientseitig ausgeführter Javascript-Code eigentlich ist: Die Browser bieten nämlich normalerweise eine Konsolen- oder Entwickler-Ansicht, mit der alle Variablen und Datenstrukturen, die sich gegenwärtig im Arbeitsspeicher befinden, eingesehen werden können. Damit ist weitgehende Transparenz über die beim Client verfügbaren Daten gegeben, auch wenn diese nicht alle tatsächlich vom Browser ausgegeben werden. 


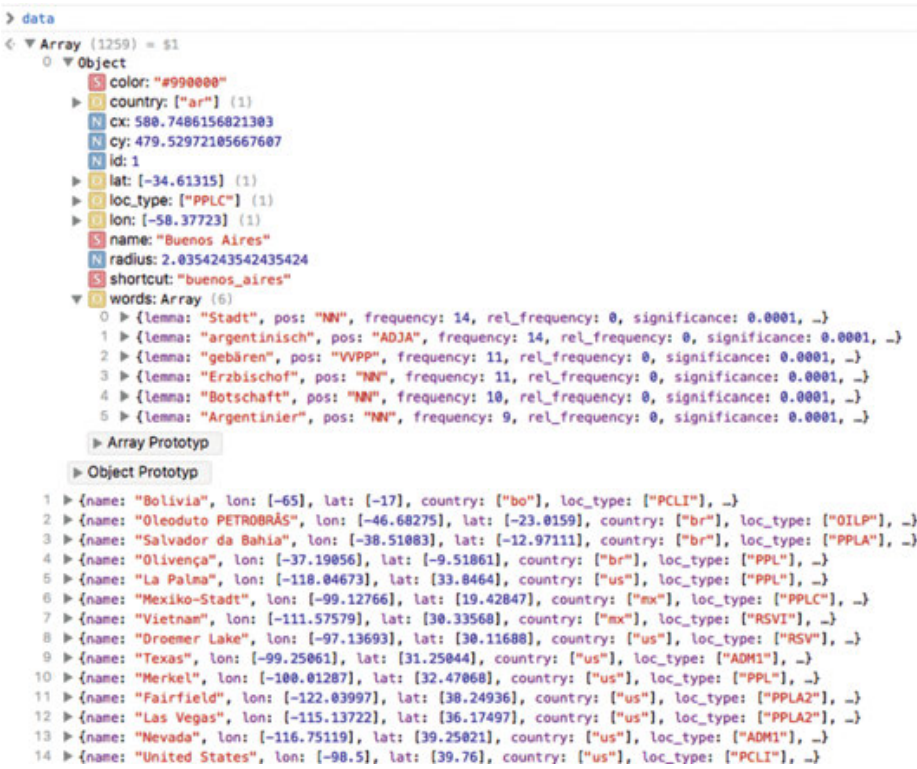

Abb. 78: Konsolenansicht im Browser (Apple Safari) bei der Nutzung der GeokollokationenAnwendung; Ausgabe der Struktur und Inhalte der Variable „data“ (Ausschnitt). Diese Ausgabe ist in jedem Client erzeugbar

In unserem Fall sind trotz der Verlagerung einiger Datenmanipulationen auf den Server die Operationen noch relativ transparent, da die Menge der zurückzugebenden Daten direkt über das Bedienfeld der Geokollokationen-Anwendung beeinflusst werden kann und sich je nach den Einstellungen dort zwar der Umfang der zurückgegebenen Daten verändert, nicht jedoch deren grundlegende Struktur.

Im Bereich der Visualisierung kam es mit der Version 2 zu folgenden Erweiterungen:

- Neuer Modus zur Darstellung der Kollokatoren: Neben der Darstellung als Liste gibt es nun die Möglichkeit, dass die Anzahl der Kollokatoren mittels entsprechend skalierter Punkte dargestellt wird (vgl. Abbildung 79, S. 234).

- Bei Berührung eines Ortspunktes erscheint eine scrollbare Liste mit allen zugeordneten Kollokatoren (sogenannter „Tooltip“), absteigend geordnet nach Signifikanz und Frequenz, wobei die Wortartenzugehörigkeit farblich codiert ist (vgl. Abbildung 80). Wenn die Kollokatoren nicht direkt auf der Karte dargestellt werden können (weil es zu unübersichtlich würde), scheint die separat aufrufbare geordnete Liste die beste Möglichkeit, sich schnell einen Überblick verschaffen zu können. 


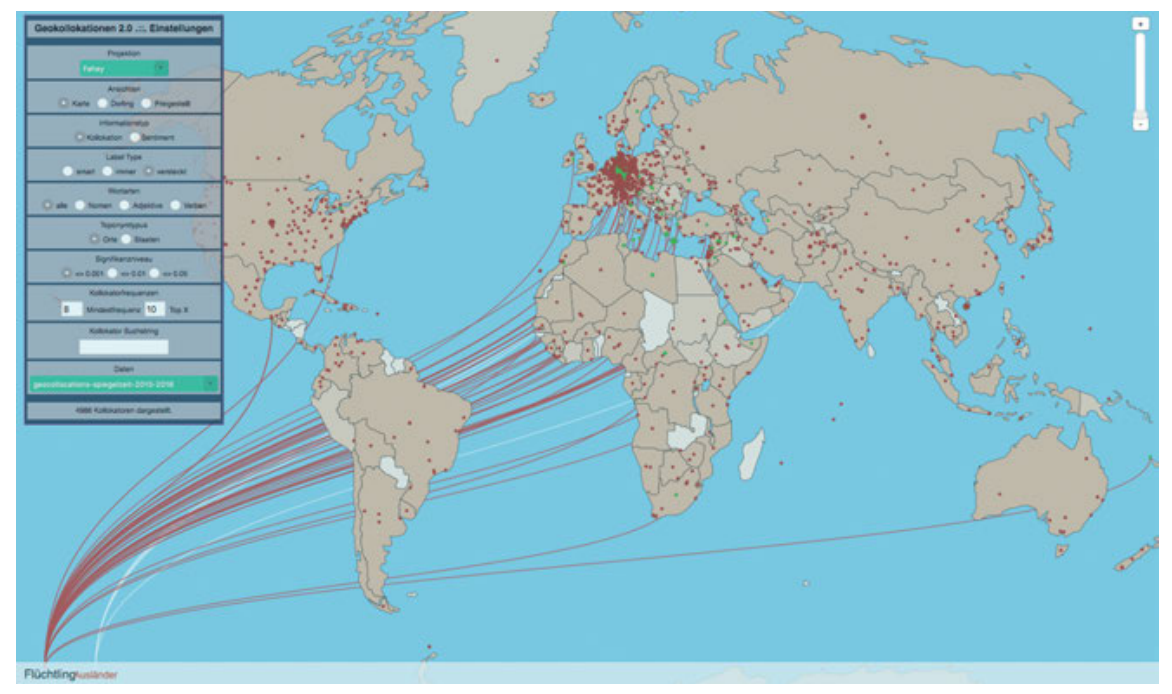

Abb. 79: Version 2 der Geokollokationen-Anwendung; Übersicht

- Über einen Rechtsklick auf einen Ortspunkt können weitere Befehle aufgerufen werden; dazu siehe die weiteren Ausführungen unten.

- Um globale und lokale Kollokatoren und generell deren Verbreitung visualisieren zu können, gibt es eine neue Darstellung, die vom sogenannten „Sankey-Diagramm“ inspiriert ist (Sankey 1896). Über das Menü mit den weiteren Befehlen kann ein Kollokator ausgewählt werden, dessen Verbreitung angezeigt werden soll. Der Kollokator wird dann am unteren Bildschirmrand aufgeführt und Linien verbinden diesen mit allen Ortspunkten, an denen er vorkommt (vgl. die Linien von „Flüchtling“ zu den Ortspunkten in Abbildung 79). Die entsprechenden Ortspunkte werden grün hervorgehoben. Die Strichdicke korreliert mit der Frequenz des Kollokators. Diese Darstellung erwies sich jedoch im sogenannten „Dorling“-Modus als nützlicher, auf den ich gleich zu sprechen komme.

- Die Datengrundlage kann über das Bedienfeld beeinflusst werden; so können wie auch schon bei der Vorversion mit Regulären Ausdrücken zur Kollokatoren mit bestimmten Zeichenfolgen dargestellt werden. 


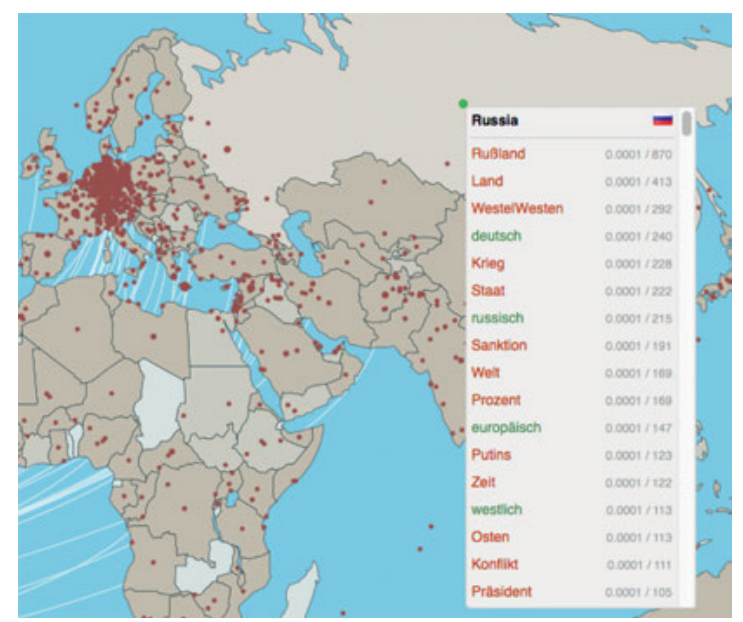

Abb. 80: Geokollokationen Version 2: Tooltip-Menü für die Anzeige der Kollokatoren

\subsubsection{Loslösung von der geographischen Darstellung}

Ein kritischer Punkt der Kartendarstellung ist ihre Bindung an die Topographie. Geht man von der Forschungsfrage nach diskursiv geprägten Konstruktionen von Welt aus, interessiert ja unter Umständen gerade die Differenz dieser zur geographisch-topographischen Weltsicht und die daraus ableitbaren Beobachtungen. Diese geographisch-topographische Weltsicht wird durch die Kartendarstellung zwar auch nur verzerrt dargestellt, da die Visualisierung der Welt auf einer zweidimensionalen Fläche nur über eine Projektion der kugelartigen Form auf die Fläche erreicht werden kann (vgl. zum Problem der Projektionen bei der Kartendarstellung und zur kritischen Kartographie Smith 1992; Glasze 2009). Doch davon abgesehen, müssten diskursiv geprägte Weltsichten nochmals weiter entfernt von der geographischen Topographie gestaltet sein.

Es gibt in der Kartographie verschiedene Ansätze, die Kartendarstellung mittels weiterer Variablen wie Bevölkerungsdichte, Wirtschaftskraft o. ä. zu verzerren. ${ }^{79}$ Eine Möglichkeit ist die sogenannte „Dorling“-Karte (Dorling 1993), bei der geographische Einheiten wie Staat, Provinz, Gemeinde o.ä. in ihren Formen bezüglich einer beliebigen Variable verzerrt dargestellt werden. Ein einfacher Algorithmus ist dabei die Überführung der Umrisse der geographischen Einheiten in Kreise. Die Größe der Kreise ist abhängig vom Wert der Variable und die

79 Vgl. etwa das „Wordmapper“-Projekt: http://www.worldmapper.org/ (22. 9. 2020). 
Kreise werden, ausgehend von ihrer ursprünglichen geographischen Position, so angeordnet, dass sie sich nicht überlappen.

Für die Geokollokationen-Anwendung ist der Gedanke naheliegend, die Ortspunkte als Kreise in Abhängigkeit der Summe der Frequenzen der signifikanten Kollokatoren dazu darzustellen. ${ }^{80}$ Dabei realisieren wir einen fließenden Übergang zwischen der konventionellen Kartendarstellung und dem Dorling-Diagramm: Die Umrisse der jeweiligen Ortspunkte werden $\mathrm{zu}$ den verschieden großen Kreisen transformiert und werden, ausgehend von der ursprünglichen Lage auf der Karte, relokalisiert, so dass sie sich gegenseitig nicht überlappen. Diese grafische Transformation ist wichtig, um die konzeptionelle Transformation der diagrammatischen Darstellung nachvollziehen und deren Ergebnis verstehen zu können (vgl. für eine Diskussion zu Animationen in Visualisierungen Fisher 2010).

Eine Schwierigkeit liegt jedoch in der Problematik, dass die Ortspunkte beliebige geografische Einheiten sein können: Städte, Gemeinden, Provinzen, Staaten, Kontinente. Es ergeben sich also sehr viele Kreise in der Dorling-Darstellung. Technisch ist das ohne Weiteres darstellbar, aber der Bezug zur ursprünglichen Karte ist noch schwächer, da beispielsweise die Städte eines Staates eigene Kreise außerhalb eines weiteren Kreises für den ganzen Staat sind. Es ist aber möglich, die Darstellung bereits bei der konventionellen Karte auf Ebene Staaten zu aggregieren, also die Kollokatoren der verschiedenen Ortspunkte innerhalb eines Staates zusammenzuführen. Dann ist der Bezug zur ursprünglichen Karte viel deutlicher.

Die Abbildungen 81 und 82 zeigen Dorling-Karten, die einen „Flucht-Diskurs“ in zwei verschiedenen Zeitabschnitten (1961-1980 und 2000-2016) repräsentieren sollen. Es werden Kollokatoren angezeigt, die auf den Regulären Ausdruck .*([Ff]l[uü]cht|[Mm]igra).* passen, also alle Derivationen der Lexeme „Flucht“ und „Migration“ / „Migrant“.

Die Karten zeigen jeweils eine deutliche Fokussierung der (in diesen spezifischen Daten repräsentierten) Diskurse auf Deutschland, der jedoch bei den neueren Daten von 2000-2016 noch viel stärker ist als in der Zeit von 1961-1980. Weiter zeigen sich klare Verschiebungen der weiteren in den Diskurs involvierten Regionen: (Eher) südliches Afrika und Asien in den älteren, Nordafrika und Naher Osten in den neueren Daten. Zwar sind auch in den älteren Daten einige europäische Länder in diesen Kontexten genannt, das ist bei den neueren Daten jedoch viel stärker ausgeprägt, vor allem jene Länder, durch die Migrationswanderungen laufen.

80 Und die Größe der Kreise sollte in Abhängigkeit der jeweils ausgewählten Datenmenge stehen, d. h., wenn über die Filtermöglichkeiten, etwa auch die Beschränkung auf bestimmte Kollokatoren, die Datenmenge eingeschränkt wird, passt sich auch die Größe der Kreise an. 


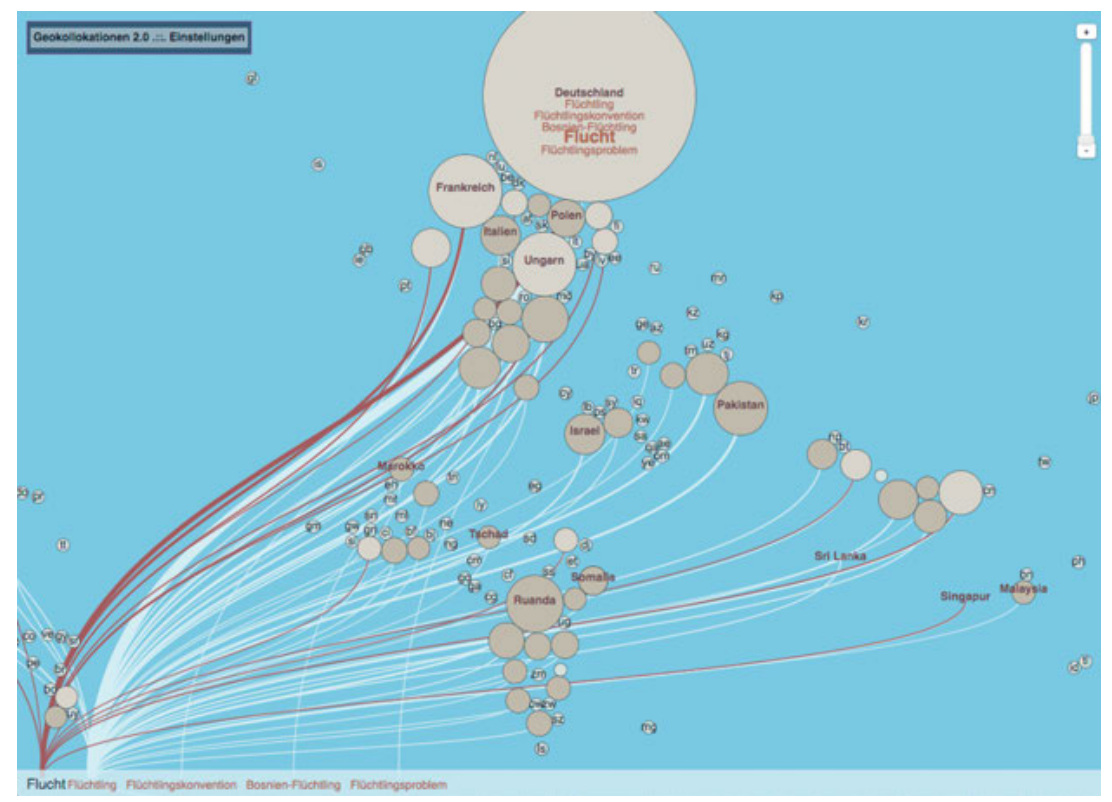

Abb. 81: Geokollokationen Dorling-Darstellung; Flucht-Diskurs 1961-1980

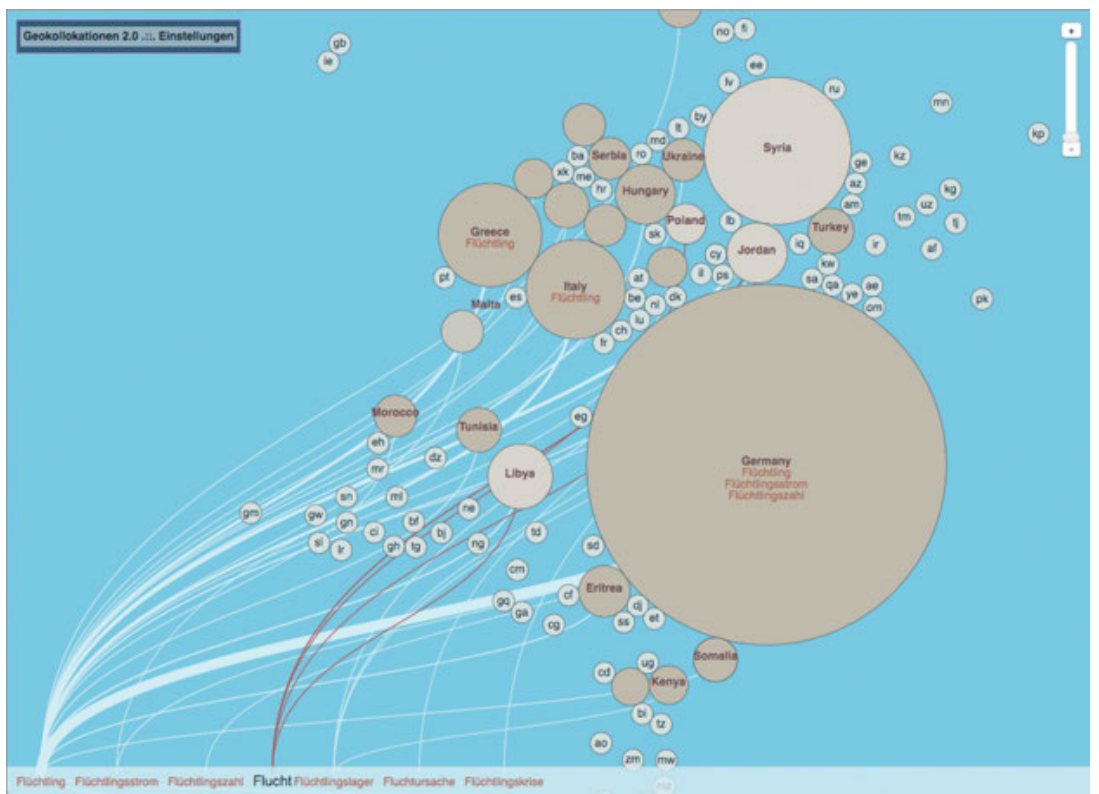

Abb. 82: Geokollokationen Dorling-Darstellung; Flucht-Diskurs 2000-2016 
Neben den möglichen feineren Deutungen, die ich hier nicht anstellen möchte, zeigt der Effekt der Dorling-Darstellung jedoch ziemlich klar den deutlichen innenpolitischen Fokus der aktuellen Migrationspolitik. Thema ist viel weniger die Frage nach den Regionen, in denen Flucht tatsächlich entsteht bzw. stattfindet, sondern Europa - und zumindest aus der Perspektive der deutschen Presse insbesondere Deutschland selbst. ${ }^{81}$

\subsection{Fazit}

Ich möchte nun die bisherige Diskussion der Geokollokationen aus diagrammatischer Perspektive prägnant zusammenfassen, um die Studie vor dem Hintergrund der erarbeiteten theoretischen Überlegungen situieren zu können. Danach soll ein Ausblick gegeben werden, wie das Visualisierungsprinzip weiter entfaltet werden kann.

\subsubsection{Diagrammatische Verortung}

Durch die topografische Deutung der Toponyme, der Georeferenzierung und der damit möglich gewordenen Kartendarstellung (diagrammatische Grundfigur: Karte) kam es zu einer Dimensionsanreicherung der Daten. Die geografische Dimension ermöglicht dadurch bei der Interpretation eine neue Sicht auf die Daten, da ihre räumliche Konstellation darstellbar wird. Gleichzeitig ist es so, dass zu den Toponymen Kollokationsprofile berechnet worden sind, um deren diskurslinguistisches Profil zu modellieren. Die Berechnung der Kollokationsprofile ist möglich, weil ein Vektorraum angenommen wird, in dem die Toponyme aufgrund ihres Kollokationsverhaltens positioniert werden können. Diese Annahme nutzt also die diagrammatische Grundfigur des Vektors aus. Mit dieser diagrammatischen Transformation gehen sowohl eine Rekontextualisierung als auch eine Desequenzialisierung der Daten einher: Die Einheit der Texte wird aufgebrochen, indem in einem ersten Schritt für die Kollokationsberechnung Indizes (diagrammatische Grundfigur: Liste) erstellt werden müssen, wobei diese selber, als auch die daraus entstehenden Kollokationsprofile die aus den Texten hinausgerissenen Einzellexeme neu kontextualisiert werden.

81 Es wäre zu prüfen, ob dieser Fokus auf die Innenpolitik bei Flüchtlingsdiskursen auch bei anderen Ländern der Fall ist und ob dies alle Aspekte des Diskurses betrifft. 
Mit der Kartendarstellung bewegt man sich in einer stark kanonisierten Visualisierungspraxis, die von bestimmten Denkstilen geprägt ist. Mit der kritischen Kartographie gibt es jedoch Ansätze, die die Praktiken in Frage stellen und beispielsweise das Problem der verzerrenden Projektionen eines dreidimensionalen Raums auf die Fläche problematisieren. Hier kommt es nun zu einer fruchtbaren Wechselwirkung zwischen programmiertechnischen Coding Cultures und Überlegungen der kritischen Kartographie: Um alternative Visualisierungsprinzipien für geografische Daten zu finden, sind Experimente wichtig. Während bis vor wenigen Jahren hochspezialisierte Software verwendet werden musste, um digitale Karten zu erstellen, entwickelte sich eine Coding Culture, die geprägt ist durch Open Source, White Box, Entprofessionalisierung und Selbstermächtigung. Mit dieser gibt es nun die technischen Möglichkeiten wie Javascript und D3, wie wir sie für unsere Arbeiten ebenfalls verwendet haben. Mit diesen Technologien kann ohne großen Aufwand und auf Basis von Beispielen Code an die eigenen Daten angepasst werden. Die Bibliotheken zur Darstellung von Karten sehen beispielsweise standardmäßig vor, dass zwischen unterschiedlichen Projektionen gewechselt werden kann. Wichtig in unserem Fall waren bestehende Bibliotheken und Programmierbeispiele jedoch für die Entwicklung der DorlingDarstellung der Karten, die ja Netzgraph, Flussdiagramm und Karte miteinander verbindet. Damit werden die bereits verwendeten diagrammatischen Grundfiguren der Liste und der Karte um die Grundfigur Graph ergänzt, womit sich der Möglichkeitsraum des diagrammatischen Operierens stark erweitert und ein reiches Visualisierungsprinzip entsteht (vgl. Abschnitt 5.7).

\subsubsection{Ausblick}

Die Ausführungen zu den Geokollokationen zeigen den langen Weg der Genese dieser Visualisierungsanwendung. Hinzu tritt der Befund, dass - sobald man damit arbeitet - weitere Ideen für Berechnungs- und Visualisierungsmöglichkeiten entstehen. Ist erst ein Prinzip der Darstellung, also ein bestimmtes Transformationsverfahren in Verbindung mit einer diagrammatischen Grundfigur, gefunden (hier die Darstellung der Geokollokationen auf verschiedenen Karten), lassen sich darin mannigfaltige Darstellungsvarianten ableiten, so dass sich ein reiches Visualisierungsprinzip mit einem weiten Möglichkeitsraum ergibt. Das scheint mir, wie oben erwähnt, bei den Geokollokationen der Fall zu sein und ich möchte diese weiteren Möglichkeiten nun folgend skizzieren.

Vorab ist aber Folgendes wichtig: Reiche Visualisierungsprinzipien lassen sich schlecht in ein abgeschlossenes Tool zwingen. Bei Präsentationen der Geokollokationenanwendung tauchten immer wieder Fragen nach der Software auf: 
Ob das Tool heruntergeladen und für eigene Daten verwendet werden kann. Zwar veröffentlichten wir den kompletten Code der Geokollokationenanwendung ${ }^{82}$, verfolgten jedoch nicht das Ziel, eine sogenannt „Out of the Box“ benutzbare Software zur Verfügung zu stellen. Und dies aus dem Grund, da wir die Erstellung der Visualisierungsprinzipien - und damit auch die Programmierung - und die Analyse der Daten als nicht zu trennendes Arbeitspaket ansehen. Die Ausführungen zur Genese der Geokollokationen-Anwendungen sollten gezeigt haben, wie technische Moden und wissenskulturelle Prägungen bestimmte programmiertechnische Umsetzungen nahelegen und man sich damit in bestimmte Coding Cultures einfügt und sich ihnen und ihren Topoi unterwirft. Damit schlägt man nicht nur bestimmte Wege der technischen Umsetzung, sondern eben auch der diagrammatischen Lösungen und des Designs ein. Das komplette Paket von digitaler Datentransformationen, algorithmisch erstellten Diagrammen und dafür genutzten Codes ist zwar mächtig in dem Sinne, dass komplexe interaktive Visualisierungen erstellt werden können, die auf großen Datenmengen basieren. Gleichzeitig ist man aber in deutlich vorgegebenen Bahnen der diagrammatischen Praxis gefangen. Dieser Bahnen der Praxis sollte man sich bei der Erstellung von Visualisierungen auf allen Ebenen und zu allen Zeitpunkten bewusst sein, was nur dann geht, wenn das Tool eine ,offene Box‘ ist und man die technische Expertise und damit den Zugriff auf alle Ebenen und Komponenten besitzt.

Innerhalb des Visualisierungsprinzips, auf dem die Geokollokationenanwendung beruht, besteht nun ein weiter Möglichkeitsraum: Insbesondere die Transformation der klassischen topografischen zur abstrakteren Dorling-Karte ist dafür verantwortlich. Auf Basis dieser Transformationen ergibt sich eine Erweiterung des Möglichkeitsraums um eine abstraktere, von der geografischen Ordnung entfernten Ebene.

Bei der Illustrierung dieser Dorlingdarstellung (vgl. Abschnitt 6.2.5) zeigte ich nur Karten, deren Bezug zur geografischen Karte noch deutlich sichtbar ist. Die Dorlingkarte erlaubt darüber hinaus aber auch alternative Gruppierungen der Länder, etwa aufgrund der Ähnlichkeit ihrer Kollokationsprofile: Länder, die ähnliche Kollokatoren aufweisen, gruppieren sich zu Clustern, so dass die Differenz zwischen diskursiv aufgeladener Semantik eines Landes mit dessen geografischen Lage deutlich hervortritt. Eine andere Möglichkeit besteht darin, die Länder gemäß Ähnlichkeit spezifischer Attribute zu clustern.

Ein Beispiel für eine Clusterung von Ländern bezüglich der Ähnlichkeit ihrer Kollokationsprofile ist die Thematisierung der Himmelsrichtungen „West“, „Ost“, „Nord“ und „Süd“ in Verbindung mit Regionen. Bei den Spiegel/Zeit-Daten

82 Vgl. https://gitlab.uzh.ch/rothenha/geocollocationsscripts (letzter Zugriff: 22. 9. 2020). 
von 2010 bis 2016 zeigt sich beispielsweise folgende Gruppierung bezüglich der Nennung von Himmelsrichtungen als Kollokator (Mindestfrequenz: 20):

- Osten: China, Indien, Afghanistan, Syrien, Israel, Russland, Ukraine, Libyen, Polen, Deutschland, Frankreich, USA

- Westen: China, Indien, Afghanistan, Iran, Syrien, Saudi-Arabien, Israel, Russland, Libyen, Deutschland, Ukraine, Polen

- Norden: Deutschland, Frankreich, Italien, Mali, Griechenland, Syrien, Afghanistan

- Süden: Deutschland, Frankreich, Italien, Spanien

Nach Ländern bezüglich ihrer Attribute gegliedert ergibt sich Tabelle 12.

Tab. 12: Ähnlichkeit von Ländern bezüglich Attribuierung mit Himmelsrichtungen

\begin{tabular}{|c|c|c|c|c|}
\hline & Osten & Westen & Norden & Süden \\
\hline Deutschland & $\mathbf{\square}$ & $\mathbf{\square}$ & $\mathbf{\square}$ & $\mathbf{\square}$ \\
\hline Afghanistan & $\mathbf{\square}$ & $\mathbf{\square}$ & $\mathbf{\square}$ & \\
\hline Syrien & $\mathbf{\square}$ & $\mathbf{\square}$ & $\mathbf{\square}$ & \\
\hline China & $\mathbf{\square}$ & $\mathbf{\square}$ & & \\
\hline Indien & $\mathbf{\square}$ & $\mathbf{\square}$ & & \\
\hline Israel & $\mathbf{\square}$ & $\mathbf{\square}$ & & \\
\hline Libyen & $\mathbf{\square}$ & $\mathbf{\square}$ & & \\
\hline Polen & $\mathbf{\square}$ & $\mathbf{\square}$ & & \\
\hline Russland & $\mathbf{\square}$ & $\mathbf{\square}$ & & \\
\hline Ukraine & 口 & $\mathbf{\square}$ & & \\
\hline Frankreich & 口 & & 口 & 口 \\
\hline USA & 口 & & & \\
\hline Iran & & 口 & & \\
\hline Saudi-Arabien & & 口 & & \\
\hline Italien & & & $\square$ & $\mathbf{\square}$ \\
\hline Griechenland & & & $\mathbf{\square}$ & \\
\hline Mali & & & $\mathbf{\square}$ & \\
\hline Spanien & & & & $\mathbf{\square}$ \\
\hline
\end{tabular}


Es gibt aus deutscher, medial repräsentierter Perspektive zwischen 2010 und 2016 eine Gruppe von Ländern, die bipolar mit Westen und Osten, sowie ein Land, Italien, das bipolar mit Norden und Süden attribuiert wird, bei anderen hingegen ist nur eine unipolare oder dann tri- oder tetrapolare Attribuierung zu beobachten. Dahinter stecken unterschiedliche semantische Lesarten der Himmelsrichtungen, nämlich einerseits eine semantisch nicht weiter konnotierte Regionenbezeichnung („die Stadt befindet sich im Norden von Frankreich“), andererseits eine ideologisch oder anderweitig aufgeladene bipolare Beziehung (,der wirtschaftlich erfolgreiche Norden hat genug vom armen Süden“ oder „die Ukraine ist Puffer im Spannungsverhältnis von Ost und West“). Dabei müssen im letzteren Fall natürlich nicht beide Himmelsrichtungen im selben Text oder gar Satz genannt werden, wie das folgende Beispiel zeigt:

Über Ethik will Yang nicht sprechen: „In der Firma redet man nur über Geschäfte.“ Mögen sich die Politiker in Peking, Washington und Brüssel mit Handelsregeln für das Textilgeschäft abmühen - China ist für ihn nicht mehr das Land der Seide, sondern eine Art wilder Osten des 21. Jahrhunderts, ein Land unbegrenzter Möglichkeiten. „Design und Qualität unserer Produkte sind bereits Weltklasse“, beschreibt Yang die Lage von Lenovo. (Georg Blume: „Wird die Welt chinesisch?“, Die Zeit, 16.6.2005)

Wenn jedoch eine geografische Entität in Texten sowohl in den Zusammenhang von Ost und West bzw. Nord und Süd gebracht wird, scheint insgesamt die ideologisch oder anderweitig aufgeladene Semantik im Spiel zu sein. In den Daten finden sich aber auch Beispiele, in denen West und Ost sogar im gleichen Satz genannt wird:

Und deshalb zieht keine andere Stadt in Indien die Zerstörungswut der Islamisten so auf sich wie die Gloriole am Arabischen Meer. Mumbai ist der Westen im Osten, Börse und Bollywood, das Finanzzentrum des Milliardenvolks und dessen Traumfabrik, für Indien ist es New York und Los Angeles in einem. Westlicher Sündenpfuhl für die einen, die von Geld, Globalisierung und der Moderne nach Art des Westens nichts wissen wollen, Schmelztiegel für die anderen, die dabei sein wollen. (Rüdiger Falksohn et al.: „Im Dreieck des Todes“, Der Spiegel, 49/2008)

Es ergibt sich nun also eine Gruppe von Ländern, nämlich China, Indien, Israel, Libyen, Polen, Russland und die Ukraine, bei der eine solche Lesart in „Spiegel“ und ,Zeit" vorherrschend ist.

Aufgrund solcher Daten, die jedoch noch sorgfältiger und weitergehender aufbereitet werden müssten, indem z. B. alle Derivationen von Nord, Süd, West und Ost berücksichtigt würden, kann eine diskursiv geprägte Weltsicht kartografiert werden. Die Dorling-Darstellung bietet die nötige Flexibilität, um dies diagrammatisch umzusetzen. 
Bei einer von der geografischen Karte stark abstrahierten Darstellung stellt sich jedoch die Frage nach ihrem Mehrwert gegenüber anderen Darstellungen, wie etwa einer Tabelle (wie Tabelle 12). Der muss in der Transformation zwischen konventioneller Karte und Dorling-Karte liegen, mit der die Reorganisation visuell wahrgenommen werden kann. Gleichzeitig könnte die Distanz zwischen geografischer und neuer Position grafisch angezeigt werden, was wiederum ein neu zu deutendes Kriterium für eine Klassifizierung zweiter Ordnung wäre.

Je nach Forschungsinteresse und interpretatorischer Methode sind weitere Ausbauten der Geokollokationenanwendung denkbar. Die Standardforderung der Visual Analytics, nach Ben Shneidermans „Visual Information Seeking Mantra“ - „Overview first, zoom and filter, then details-on-demand“ (Shneiderman 1996) - würde eine engere Verknüpfung der dargestellten Geokollokationen mit den Originaltexten vorsehen. Damit gesellt sich zur diagrammatischen Grundfigur der Karte noch jene der Liste als Index für die Volltexte, was den Möglichkeitsraum des Visualisierungsprinzips nochmals stark erweitert.

Die Ausführungen zu den Geokollokationen möchte ich an dieser Stelle beenden. Ich hoffe gezeigt zu haben, wie stark durchdrungen von technologischen, methodologischen und wissenschaftskulturellen Fragen und Bedingungen die Entwicklung einer Visualisierung ist und dass es sich lohnt, diese Aspekte immer wieder zu reflektieren. 